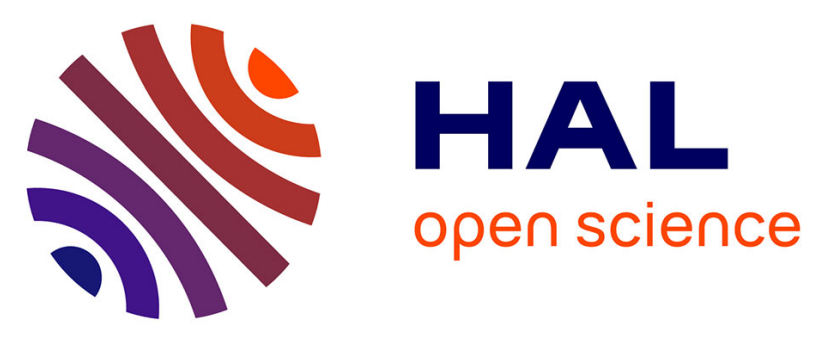

\title{
Second-order autoregressive model-based Kalman filter for the estimation of a slow fading channel described by the Clarke model: Optimal tuning and interpretation
} Ali Houssam El Husseini, Eric Pierre Simon, Laurent Ros

\section{- To cite this version:}

Ali Houssam El Husseini, Eric Pierre Simon, Laurent Ros. Second-order autoregressive model-based Kalman filter for the estimation of a slow fading channel described by the Clarke model: Optimal tuning and interpretation. Digital Signal Processing, 2019, 90, pp.125-141. 10.1016/j.dsp.2019.04.008 . hal-02112635

\section{HAL Id: hal-02112635 \\ https://hal.science/hal-02112635}

Submitted on 26 Apr 2019

HAL is a multi-disciplinary open access archive for the deposit and dissemination of scientific research documents, whether they are published or not. The documents may come from teaching and research institutions in France or abroad, or from public or private research centers.
L'archive ouverte pluridisciplinaire HAL, est destinée au dépôt et à la diffusion de documents scientifiques de niveau recherche, publiés ou non, émanant des établissements d'enseignement et de recherche français ou étrangers, des laboratoires publics ou privés. 


\title{
Second-order autoregressive model-based Kalman filter for the estimation of a slow fading channel described by the Clarke model: Optimal tuning and interpretation ${ }^{2}$.
}

\author{
Ali Houssam El Husseini ${ }^{\mathrm{a}}$, Eric Pierre Simon ${ }^{\mathrm{a}}$, Laurent Ros ${ }^{\mathrm{b}}$ \\ ${ }^{a}$ University of Lille, UMR 8520 - IEMN, F-59655 Villeneuve d'Ascq, France \\ ${ }^{b}$ Univ. Grenoble Alpes, CNRS Grenoble INP, 的该, GIPSA-lab, 38000, Grenoble, France
}

\section{Abstract}

This paper treats the estimation of a flat fading Rayleigh channel with Jakes' Doppler spectrum model and slow fading variations. A common method is to use a Kalman filter (KF) based on an auto-regressive model of order $p$ $(\operatorname{AR}(p))$. The parameters of the AR model can be simply tuned by using the correlation matching $(\mathrm{CM})$ criterion. However, the major drawback of this method is that high orders are required to approach the Bayesian CramerRao lower bound. The choice of $p$ together with the tuning of the model parameters is thus critical, and a tradeoff must be found between the numerical complexity and the performance. The reasonable tradeoff arising from setting $p=2$ has received much attention in the literature. However, the methods proposed for tuning the model parameters are either based on an extensive grid-search analysis or experimental results, which limits their ap-

\footnotetext{
${ }^{\text {¿ }}$ Part of this paper was presented in the conference paper [1].

wistitute of Engineering Univ. Grenoble Alpes Email addresses: ali.el-husseini@univ-lille.fr (Ali Houssam El Husseini), eric.simon@univ-lille.fr (Eric Pierre Simon), laurent.ros@gipsa-lab.grenoble-inp.fr (Laurent Ros)
} 
plicability. A general solution for any scenario is simply missing for $p=2$ and this paper aims at filling this gap. We propose using a Minimization of Asymptotic Variance (MAV) criterion, for which a general closed-form formula has been derived for the optimal tuning of the model and the mean square error. This provides deeper insight into the behaviour of the KF with respect to the channel state (Doppler frequency and signal to noise ratio). Moreover, the paper interprets the proposed solution, especially the dependence of the shape of the optimal $\operatorname{AR}(2)$ spectrum on the channel state. Analytic and numerical comparisons with first- and second-order algorithms in the literature are also performed. Simulation results show that the proposed AR(2)-MAV model performs better than the literature and similarly to $\mathrm{AR}(p)$-CM models with $p \geq 15$.

\section{Introduction}

This paper treats the estimation of a flat fading channel in the context of slow fading, i.e. normalized Doppler frequencies less than $10^{-2}$. Note that this context includes a large number of practical applications, including vehicular applications. For instance, with the vehicular communication standard $802.11 \mathrm{p}$ [2], this corresponds to a speed of hundreds of $\mathrm{km} / \mathrm{h}(228 \mathrm{~km} / \mathrm{h})$. The

principle of this channel estimation is the tracking of the complex baseband equivalent flat fading coefficient, called the channel complex gain (CG), which will be denoted by $\alpha$. Here, the widely accepted Rayleigh random model with Jakes' Doppler spectrum (a model proposed by Clarke in 1968 [3]) will be employed. Common approaches to channel estimation are to employ adaptive filters, such as least mean square (LMS) and Kalman filters (KF). The 
LMS is a computationally less demanding technique than KF, but at the cost of a slower convergence. The design of KF requires selecting a linear recursive state-space model for the parameter to be tracked [4 8]. As the true CG does not follow such a model, the linear recursive state-space model is considered an approximation. Thus, it has to be selected and tuned carefully in order to limit any degradation. The conventional state-space model is an autoregressive model of some order $p(\operatorname{AR}(p))[9$, 10, whose parameters are tuned by matching the autocorrelation of the true channel CG with that of the AR process. This criterion is known as the correlation matching (CM) criterion [9, 11 13 1 $]^{1}$ and the solution is obtained by solving the YuleWalker equations [14]. Over the past two decades, an extensive literature on Rayleigh Channel estimation was based on an $\operatorname{AR}(p)$-KF tuned using the CM criterion [12, 15-18, 11] and still continues nowadays [19 24].

\subsection{Challenge of slow fading channel estimation with an AR(p)-Kalman fil- ter of low order}

In the context of very high mobility, such as high speed trains, [25, 26] used an $\operatorname{AR}(p)-\mathrm{KF}$ of order $p=1$ tuned with the $\mathrm{CM}$ criterion. Simulation results show outstanding performance in such scenarios in terms of MSE. In the most common context of a slow fading scenario, the use of a CM criterion with an $\operatorname{AR}(p)-\mathrm{KF}$ of low order ( $p=1$ or 2$)$ for Clarke model channel estimation was also usual up to ten years ago [12, 15, 16, 13]. However, those papers

\footnotetext{
${ }^{1}$ Note that this is one of the methods used in Matlab to approximate correlated fading channels for the computer simulation of the Rayleigh fading channel model with Jakes' Doppler spectrum.
} 
do not compare the MSE to lower bounds such as the Bayesian Cramer-Rao Bound (BCRB). Abeida et al. [27] make this comparison and note an MSE very far from the modified CRB (see [27] fig. 3, with $f_{d} T=7.38 \times 10^{-4}$, $p=1$ ), arguing that the CRB is not achievable in a low SNR region. But the right explanation, due to Ghandour-Haidar et al. 28] in 2012, after the preliminary work of Barbieri et al. [29] in 2009, is that the CM criterion with low order $p$ is not able to follow the dynamics of the fading, since the dynamic MSE is approximately constant with respect to the Doppler frequency (see eq. (22) or fig. B.1 in [28]), whereas it should be able to decrease for low Doppler (the channel is theoretically easier to estimate). In conclusion, disappointing performance is obtained with low-order $\operatorname{AR}(p)-\mathrm{KF}$ tuned with the $\mathrm{CM}$ criterion for the slow fading scenario, which is the most common one for practical applications, and solutions have to be found. It should be noted that in spite of this important warning highlighted from 2012, many authors still continue to use the KF with an AR ( $p=1$ or 2 ) model tuned with the CM criterion for Clarke channel estimation [19 24] with low to moderate Doppler frequencies. It appears thus important to continue to communicate on this point, and recommend alternative solutions.

\subsection{Existing techniques, limitations, and open questions}

In [9] and [18] there is proposed a correction of the CM criterion by the addition of a very small regularization term $\epsilon$ to the diagonal of the correlation matrix, to enhance its conditioning. This correction considerably improves the performance, but the parameter $\epsilon$ is only set by simulation and high orders are still required in order to get closer to the BCRB, as can be seen in Fig. 1, where the MSE performance for the $\operatorname{AR}(p)-\mathrm{CM}+\epsilon$ is presented. Thus, 


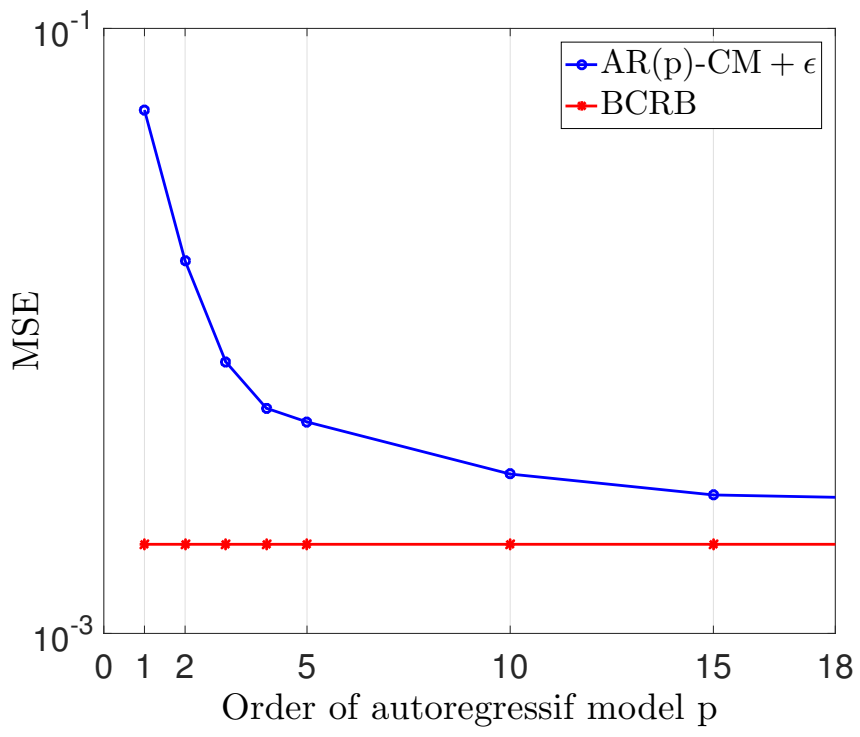

Figure 1: Performance in terms of MSE of the $\mathrm{AR}(p)-\mathrm{CM}+\epsilon$ where $\epsilon$ is set according to 9] for $f_{d} T=10^{-3}$ and SNR $=10 \mathrm{~dB}$.

the choice of the order together with the tuning of the model parameters is critical and a trade-off must be found between numerical complexity and performance. The case $p=1$ was fully resolved in [28, 29], but it leaves room for possible improvement regarding the BCRB. The reasonable trade-off arising from $p=2$, when considering a tuning other than the CM, has received much attention in the literature, but without a satisfactory analytical solution, as detailed below.

The $\operatorname{AR}(2)$ model has one pair of parameters to be tuned: the coefficients $\left\{a_{1}, a_{2}\right\}$ of the linear difference equation, or, equivalently, the resonance frequency and the pole radius $\left\{f_{\mathrm{AR}(2)}, r\right\}$. [30] provides analytical tuning for only one parameter, $f_{\mathrm{AR}(2)}$, while the second parameter $r$ is fixed through intensive simulations with a search grid. Similarly, [31, 4, 32] use the same 
tuning for $f_{\mathrm{AR}(2)}$ and additionally give an analytical expression for $r$ via experimentation in terms of the Doppler frequency without considering the signal to noise ratio (SNR). In [29], $\left\{a_{1}, a_{2}\right\}$ depends on a set of coefficients that have to be evaluated off-line using look-up tables from simulation results for a range of values of the SNR. Consequently, these solutions work well only in the specific context for which they have been tuned, and a general analytical solution for the tuning of the $\operatorname{AR}(2)$ model, that will work in any scenario, is still missing.

Recently, an alternative model, the random walk (RW) model, with a minimum asymptotic variance (MAV) criterion, has been employed for KF channel estimation [33, 34]. The RW model is also a Gauss-Markov model (when $p=1$, or an integrated version if $p>1)$ but unlike the $\operatorname{AR}(p)$ model, the $\mathrm{RW}(p)$ model is not stationary: it has a variance that grows to infinity with the number of iterations, and it is why it is most often used for the (modulo$2 \pi)$ phase estimation problem. Analytic solutions for the tuning of the RW $(p)$ model have been found for $p=1$ [35], $p=2$ [33] and $p=3$ [36]. We can conclude from these studies that the MSE performance of the $\mathrm{RW}(p)-\mathrm{KF}$ is close to the BCRB for $p \geq 2$.

The questions that now arise are: Is it also theoretically possible to obtain a performance close to the $\mathrm{BCRB}$ with the $\mathrm{AR}(2)$ model? Is this performance equivalent to or better than what was obtained with the RW(2) model? In addition to being open questions that deserve better answers than those that can be found in the literature, there would be an advantage to working with a stationary model, which the RW(2) model is not. The answers to these questions will be given in this paper. 
Note that part of the results of the present paper was presented in the conference paper [1]. However, [1] followed a sub-optimal approach that was slightly different: the adjustment of the parameter $a_{1}$ imposed a linear constraint with the Doppler frequency for the parameter $a_{2}$ deduced from grid search. Furthermore, no complete proof, interpretation, or extensive comparison was provided.

\subsection{Contributions of this paper}

In this paper, a novel optimal tuning of an $\mathrm{AR}(2)$ model with Kalman filter is proposed to achieve better performance in terms of MSE channel estimation in radio mobile communications with a flat fading scenario, assuming a Clarke model. To sum up, the contributions are as follows:

- Optimal tuning of an $\mathrm{AR}(2)$ model under the MAV criterion. Closedform expressions for the tuning of the parameters are provided as functions of the channel state (Doppler frequency and SNR), which make them highly useful in practice. Moreover, an expression for the optimal MSE is provided, which is useful to predict the performance of the channel estimation, also with respect to the channel state. To do so, the Riccati equations of the asymptotic KF are solved by resorting to an eigenvalue based method commonly used in optimal linear control 37.

- New interpretations of the power spectral density (PSD) of the optimal $\mathrm{AR}(2)$ process are also provided. More precisely, a closed-form expres- 
sion for the damping ratio sheds light on the shape of the PSD. Unlike classical parametric modeling, where the goal is to fit the PSD of an AR process to the true PSD, here the PSD of the optimal AR process is not necessarily the one that best fits the true PSD. We demonstrate that this is particularly true with harsh channel states, i.e. relatively high Doppler frequencies (still under the slow fading assumption) and low SNR. This is understandable, since our criterion is an optimal estimation rather than a modeling criterion. In addition, interpretations of the autocorrelation functions are also provided.

- An extensive and fair comparison with the algorithms commonly used in the literature for tracking time-varying flat fading channels is provided, from theoretical formulas or numerical simulations. The considered algorithms are Kalman filters based on an $\operatorname{AR}(p)$ model with CM [11-13] and improved criterion [4, 31, 28], or based on an RW $(p)$ model [33], but also least mean square (LMS) algorithms [35, 38, 39] or rather their integrated versions [40, 41] to have the same model order $p=2$ as the proposed algorithm.

\subsection{Outline of this paper}

The rest of this paper is organized as follows. Section 2 provides an introduction to the system model. In this section, the true channel model is given, then the approximate channel model using the $\mathrm{AR}(2)$ and the equivalent pairs of parameters of interest are presented. Section 3 presents the Kalman filter equation, the Kalman filter in steady-state, and analytic expressions 
of the Kalman filter gain, which will be useful in the optimization process. The variance of the MSE in steady-state and the optimization are given in Section 4. Interpretations and simulation results are provided in Section 5. Section 6 concludes the paper.

\section{System Model}

\subsection{True channel model}

We consider the estimation of a flat Rayleigh fading channel. The observation is 2

$$
y_{(k)}=\alpha_{(k)}+w_{(k)},
$$

where $k$ is the time index, $w_{(k)}$ is a zero-mean additive white circular complex Gaussian noise with variance $\sigma_{w}^{2}$, and $\alpha_{(k)}$ is a zero-mean correlated circular complex Gaussian channel gain with variance $\sigma_{\alpha}^{2}$. The signal to noise ratio is $\mathrm{SNR}=\sigma_{\alpha}^{2} / \sigma_{w}^{2}$ and the normalized Doppler frequency of this channel is $f_{d} T$, where $T$ is the symbol period. In this paper, slow fading is assumed, i.e. $f_{d} T \ll 1$. This assumption corresponds to many transmission scenarios, e.g. 802.11p [2], where the carrier frequency is around $5.9 \mathrm{GHz}$ and the symbol period is $8 \mu \mathrm{s}$. As $f_{d} T$ is proportional to the symbol period $T$, values around $10^{-2}$ can correspond to a relatively high mobility with such systems (hundreds of $\mathrm{km} / \mathrm{h}$ ). This prompts the need for a comprehensive study of

\footnotetext{
${ }^{2}$ Model (1) assumes that symbols are normalized and known (or decided), in addition to the flat fading assumption. Although this model is admittedly simplistic, it can be applied to different (more involved) contexts, such as pilot-aided multi-carrier systems in frequency-selective wireless channels 42 .
} 


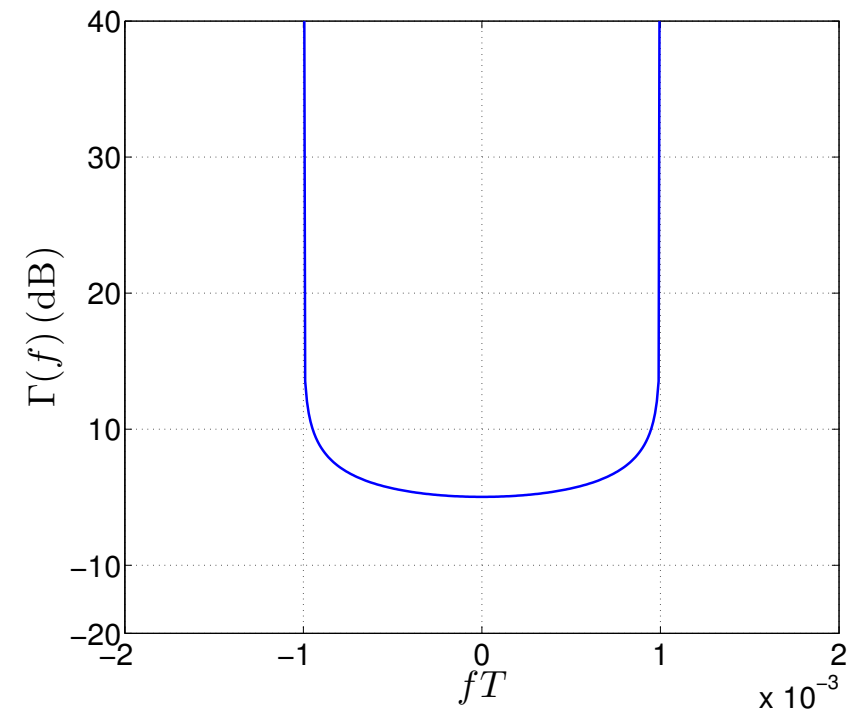

Figure 2: Centred Jakes' PSD for $f_{d} T=10^{-3}$.

channel estimation for $f_{d} T \leq 10^{-2} \ll 1$. The Jakes' Doppler spectrum (illustrated in Fig. 2) for this channel is

$$
\Gamma(f)= \begin{cases}\frac{\sigma_{\alpha}^{2}}{\pi f_{d} \sqrt{1-\left(\frac{f}{f_{d}}\right)^{2}}} & \text { if }|f|<f_{d} . \\ 0 & \text { if }|f|>f_{d} .\end{cases}
$$

It has two infinite peaks when $f$ tends to $f_{d}$ and $-f_{d}$. The autocorrelation coefficient $R_{\alpha}[m]$ of the stationary channel CG $\alpha_{(k)}$ is defined for a lag $m$ by

$$
R_{\alpha}[m]=E\left\{\alpha_{(k)} \cdot \alpha_{(k-m)}^{*}\right\}=\sigma_{\alpha}^{2} J_{0}\left(2 \pi f_{d} T m\right),
$$

where $J_{0}$ is the zeroth-order Bessel function of the first kind. 


\subsection{Approximate channel model}

In this article, the true channel $\mathrm{CG}$ is approximated by an $\mathrm{AR}(2)$ process denoted by $\tilde{\alpha}_{(k)}$ :

$$
\tilde{\alpha}_{(k)}=a_{1} \tilde{\alpha}_{(k-1)}+a_{2} \tilde{\alpha}_{(k-2)}+u_{(k)},
$$

where $u_{(k)}$ is a complex white circular Gaussian state noise with variance $\sigma_{u}^{2}$. The possible range of the real parameters of the $\operatorname{AR}(2)$ are $a_{1} \in[0,2[$ and $\left.a_{2} \in\right]-1,0[$ to ensure stationarity. Moreover, to approximate the Jakes' Doppler spectrum in the slow fading scenario, $a_{1}$ and $a_{2}$ will be necessarily close to 2 and -1 , respectively (as will be deduced later from (11)).

Assume that $\tilde{\alpha}_{(k)}$ has the same power as $\alpha_{(k)}$, i.e. $R_{\tilde{\alpha}}[0]=\sigma_{\alpha}^{2}$. The YuleWalker equations are [14]

$$
\begin{aligned}
R_{\tilde{\alpha}}[1] & =\frac{a_{1} R_{\tilde{\alpha}}[0]}{1-a_{2}} \\
R_{\tilde{\alpha}}[2] & =a_{1} R_{\tilde{\alpha}}[1]+a_{2} R_{\tilde{\alpha}}[0] \\
\sigma_{u}^{2} & =R_{\tilde{\alpha}}[0]-a_{1} R_{\tilde{\alpha}}[1]-a_{2} R_{\tilde{\alpha}}[2] .
\end{aligned}
$$

Using (5), (6) and (7) also gives $\sigma_{u}^{2}$ as a function of $a_{1}$ and $a_{2}$ alone, which will be useful for the following:

$$
\sigma_{u}^{2}=\sigma_{\alpha}^{2} \frac{\left(1+a_{2}\right)\left(1-a_{1}-a_{2}\right)\left(1+a_{1}-a_{2}\right)}{\left(1-a_{2}\right)} .
$$

Note that the CM solution for the tuning of $a_{1}$ and $a_{2}$ is obtained from (5) and (6) by imposing $R_{\tilde{\alpha}}[p]=R_{\alpha}[p]$ for $p=\{0,1,2\}$.

\subsection{Equivalent pairs of parameters of interest for $\left\{a_{1}, a_{2}\right\}$}

So far, one pair of parameters $\left\{a_{1}, a_{2}\right\}$ has been presented. Now, two equivalent pairs of parameters will also be considered. The optimal tuning will 
be investigated with the second pair, while the third pair will be used for purposes of interpretation.

\subsubsection{Parameters $\left\{f_{A R(2)} T, r\right\}$}

The pair of parameter $\left\{f_{\mathrm{AR}(2)} T, r\right\}$, equivalent to $\left\{a_{1}, a_{2}\right\}$, is derived from the transfer function of the $\operatorname{AR}(2)$ model, obtained from the $z$-transform of (4):

$$
H(z)=\frac{1}{1-a_{1} z^{-1}-a_{2} z^{-2}} .
$$

In order to design a low-pass filter, a set of complex conjugate poles should be placed in the $z$-plane at $z_{1}=r \cdot \mathrm{e}^{-j 2 \pi f_{\mathrm{AR}(2)} T}$ and $z_{2}=r \cdot \mathrm{e}^{+j 2 \pi f_{\mathrm{AR}(2)} T}$ [4, 30]:

$$
H(z)=\frac{1}{\left(1-z_{1} z^{-1}\right)\left(1-z_{2} z^{-1}\right)}
$$

Comparing Eqs (9) and (10), we have

$$
a_{1}=2 r \cos \left(2 \pi f_{\mathrm{AR}(2)} T\right) \quad a_{2}=-r^{2},
$$

where $r \in\left[0,1\right.$ is the radius of the poles, and $f_{\mathrm{AR}(2)} T \in[0,1[$ is the normalized resonance frequency of the $\operatorname{AR}(2)$ process.

In order to obtain a resonance peak, it is well known that $r$ must be close to 1 . Put $\delta=1-r$. This leads to the assumption $0<\delta \ll 1$, which will be exploited to get simple approximate closed-form expressions. Then, to position the peak at $f_{d} T$, the resonance frequency $f_{\mathrm{AR}(2)} T$ must be around $f_{d} T$, yielding $f_{\mathrm{AR}(2)} T \ll 1$. Initially, $f_{\mathrm{AR}(2)} T$ was fixed to $f_{d} T$ [4]. Then, [31] showed that the best choice for the Kalman estimation is actually

$$
f_{\mathrm{AR}(2)} T=\frac{1}{\sqrt{2}} f_{d} T
$$




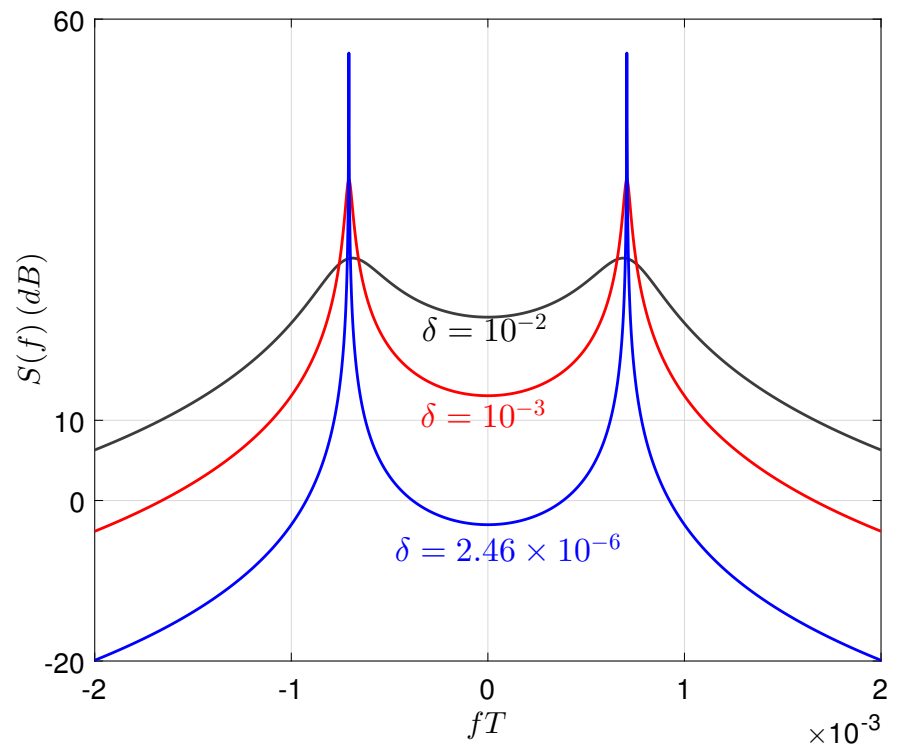

Figure 3: An example of PSDs for an $\operatorname{AR}(2)$ for $f_{d} T=10^{-3}$ with $f_{\mathrm{AR}(2)} T=\frac{f_{d} T}{\sqrt{2}}$ and different values of $\delta=1-r$.

The compensation factor $\sqrt{2}$ has also been justified in [30]. Assumption (12) is adopted a priori in this article, and will be in addition verified in Fig. 5 through simulations. However, in [30, 31], no expression is given for the tuning of $r$. The optimal tuning of $r$ is the topic of the next sections. To end this subsection, an example of the PSD of the $\mathrm{AR}(2)$ process $S(f)=\sigma_{u}^{2}\left|H\left(\mathrm{e}^{j 2 \pi f T}\right)\right|^{2}$ is given in Fig. 3 for three different values of $\delta$, thus illustrating the connection between the value of $\delta$ and the height of the peaks.

\subsubsection{Parameters $\left\{f_{n, A R(2)} T, \zeta_{A R(2)}\right\}$}

The equivalent natural frequency $f_{n, \operatorname{AR}(2)} T$ and damping ratio $\zeta_{\mathrm{AR}(2)}$ of the discrete-time second-order low-pass filter $H(z)$ could also be investigated. 
These parameters are indeed the most used to tune second order filters, in the case of continuous-time systems [43, 34]. The following notation will be used in the following sections: $\omega_{\mathrm{AR}(2)}=2 \pi f_{\mathrm{AR}(2)}$ and $\omega_{n, \mathrm{AR}(2)}=2 \pi f_{n, \mathrm{AR}(2)}$. Even though we will tune the $\mathrm{AR}(2)$ process with $\left\{f_{\mathrm{AR}(2)} T, r\right\}$, this third pair of positive parameters $\left\{f_{n, \operatorname{AR}(2)} T, \zeta_{\mathrm{AR}(2)}\right\}$ is of interest due to its physical meaning and we will refer to it for purposes of interpretation. Indeed, it is known that the height of the PSD peaks is inversely proportional to $\zeta_{\operatorname{AR}(2)}$, actually just like $\delta$. So it is legitimate to ask about the link between these two pairs of parameters. This is found in Appendix A.1, where we prove the following relations between $\left\{f_{n, \operatorname{AR}(2)} T, \zeta_{\mathrm{AR}(2)}\right\}$ and $\left\{f_{\mathrm{AR}(2)} T, r\right\}$ :

$$
\begin{aligned}
f_{n, \mathrm{AR}(2)} T=\frac{1}{2 \pi} & \sqrt{\left(2 \pi f_{\mathrm{AR}(2)} T\right)^{2}+\ln (r)^{2}} \\
\zeta_{\mathrm{AR}(2)} & =\frac{-\ln (r)}{2 \pi f_{n, \mathrm{AR}(2)} T} \\
& \simeq \frac{\delta}{\omega_{\mathrm{AR}(2)}} .
\end{aligned}
$$

The transition from (14) to (15) is detailed in Appendix A.2. With this formula, we show, as expected, that the coefficient $\delta$ of the $\operatorname{AR}(2)$ process is proportional to the damping ratio, but that it is also proportional to the resonance frequency of an equivalent second-order analog system.

Note that as already stated, the damping ratio should be $\zeta_{\operatorname{AR}(2)} \ll 1$. Then the formula $f_{n, \mathrm{AR}(2)} T=\frac{f_{\mathrm{AR}(2)} T}{\sqrt{1-\zeta_{\mathrm{AR}(2)}^{2}}}$, obtained from A.4 , becomes

$$
f_{n, \mathrm{AR}(2)} T \simeq f_{\mathrm{AR}(2)} T .
$$

Hence, the resonance frequency of the $\operatorname{AR}(2)$ process and the natural frequency of the equivalent second-order system have (almost) the same value. 


\section{The Kalman Filter}

First of all, recall that we chose the MAV criterion, which consists in minimizing the mean square error (MSE) in steady state mode $(k \rightarrow+\infty)$, $\sigma_{\epsilon}^{2}=E\left\{\left|\alpha_{(k)}-\hat{\alpha}_{(k \mid k)}\right|^{2}\right\}$, where $\hat{\alpha}_{(k \mid k)}$ is the estimate of $\alpha_{(k)}$ given by the Kalman filter, as detailed in Section 3.1. Then, to implement this criterion, an expression for the steady state Kalman gain will be found in Section 3.2. An approximate version, more tractable for the optimization, will be provided in Section 3.3, together with the list of the assumptions that allow us to derive it. All these assumptions match the specified context of slow fading and SNR greater than one, which is in general the case in practice.

\subsection{The equations of the Kalman filter}

The second order autoregressive model can be reformulated as a state space model model. The state vector to be considered includes the channel CG at $k$ and $k-1, \boldsymbol{\alpha}_{(k)}=\left[\alpha_{(k)}, \alpha_{(k-1)}\right]^{T}$ and $\tilde{\boldsymbol{\alpha}}_{(k)}=\left[\tilde{\alpha}_{(k)}, \tilde{\alpha}_{(k-1)}\right]^{T}$. The state transition matrix is $\mathbf{M}=\left[\begin{array}{cc}a_{1} & a_{2} \\ 1 & 0\end{array}\right]$ and the state noise vector is $\mathbf{u}_{(k)}=$ $\left[u_{(k)}, 0\right]^{T}$. The selection vector has dimensions $1 \times 2$ and is given by $\mathbf{s}^{T}=[1,0]$. The state evolution of (4) and observation (1) becomes

$$
\begin{gathered}
\tilde{\boldsymbol{\alpha}}_{(k)}=\mathbf{M} \tilde{\boldsymbol{\alpha}}_{(k-1)}+\mathbf{u}_{(k)} \\
y_{(k)}=\mathbf{s}^{T} \boldsymbol{\alpha}_{(k)}+w_{(k)} .
\end{gathered}
$$

Now we define the prediction vector $\hat{\boldsymbol{\alpha}}_{(k \mid k-1)}=\left[\hat{\alpha}_{(k \mid k-1)}, \hat{\alpha}_{(k-1 \mid k-1)}\right]^{T}$ and the estimation vector $\hat{\boldsymbol{\alpha}}_{(k \mid k)}=\left[\hat{\alpha}_{(k \mid k)}, \hat{\alpha}_{(k-1 \mid k)}\right]^{T}$, with $\hat{\alpha}_{(k \mid j)}$ being the estimate of the $\mathrm{CG}$ at time $k$ given the observation at time $j$. Regarding the state-space formulation (17) and (18), the two stages of the filter are 


\section{Time update equations:}

$$
\begin{gathered}
\hat{\boldsymbol{\alpha}}_{(k \mid k-1)}=\mathbf{M} \hat{\boldsymbol{\alpha}}_{(k-1 \mid k-1)} \\
\mathbf{P}_{(k \mid k-1)}=\mathbf{M} \mathbf{P}_{(k-1 \mid k-1)} \mathbf{M}^{H}+\mathbf{U}
\end{gathered}
$$

\section{Measurement update equations}

$$
\begin{gathered}
\mathbf{K}_{(k)}=\frac{\mathbf{P}_{(k \mid k-1)} \mathbf{s}}{\mathbf{s}^{T} \mathbf{P}_{(k \mid k-1)} \mathbf{s}+\sigma_{w}^{2}} \\
\hat{\boldsymbol{\alpha}}_{(k \mid k)}=\hat{\boldsymbol{\alpha}}_{(k \mid k-1)}+\mathbf{K}_{(k)}\left(y_{(k)}-\mathbf{s}^{T} \hat{\boldsymbol{\alpha}}_{(k \mid k-1)}\right) \\
\mathbf{P}_{(k \mid k)}=\left(\mathbf{I}_{2}-\mathbf{K}_{(k)} \mathbf{s}^{T}\right) \mathbf{P}_{(k \mid k-1)},
\end{gathered}
$$

where $\mathbf{K}_{(k)}=\left[\begin{array}{c}K_{1(k)} \\ K_{2(k)}\end{array}\right]$ is the Kalman gain vector, $\mathbf{U}=\left[\begin{array}{cc}\sigma_{u}^{2} & 0 \\ 0 & 0\end{array}\right], \mathbf{I}_{2}$ is the $2 \times 2$ identity matrix, and $\mathbf{P}_{(k \mid k)}$ and $\mathbf{P}_{(k \mid k-1)}$ are, respectively, the $2 \times 2$ a posteriori and the predicted error covariance matrices.

\subsection{Steady-state Kalman filter}

Since the linear system is observable and controllable, an asymptotic regime for which the covariances and gain of the filter become constant is quickly reached:

$\mathbf{K}_{(k)}=\mathbf{K}_{(k+1)}=\mathbf{K}_{\infty}=\left[\begin{array}{c}K_{1} \\ K_{2}\end{array}\right], \mathbf{P}_{(k \mid k)}=\mathbf{P}_{(k+1 \mid k+1)}=\mathbf{P}_{\infty}=\left[\begin{array}{cc}P_{11} & P_{12} \\ P_{21} & P_{22}\end{array}\right]$ and 
$\mathbf{P}_{(k \mid k-1)}=\mathbf{P}_{(k+1 \mid k)}=\mathbf{P}_{\infty}^{\prime}=\left[\begin{array}{cc}P_{11}^{\prime} & P_{12}^{\prime} \\ P_{21}^{\prime} & P_{22}^{\prime}\end{array}\right]$.

Note that once the KF attains the steady state, it becomes a fixed coefficient filter. After the convergence, the total complexity is reduced to the number of multiplications required at each iteration for the computation of $\hat{\boldsymbol{\alpha}}_{(k \mid k-1)}$ and $\hat{\boldsymbol{\alpha}}_{(k-1 \mid k-1)}$. The total complexity of the steady-state $\operatorname{AR}(p)-\mathrm{KF}$ is $\left(3 p^{2}+2 p\right)$ multiplications, where $p=2$ here, versus 1 multiplication for the LMS. It will be seen in Section 4 that analytical expressions for $K_{1}$ and $K_{2}$ as functions of the $\operatorname{AR}(2)$ parameters $a_{1}, a_{2}, \sigma_{u}^{2}$ and the observation noise variance $\sigma_{w}^{2}$ are required for tuning $r$. First, we focus on $K_{1}$, and $K_{2}$ will be deduced from $K_{1}$. To find $K_{1}$, we need to solve the Riccati equations, which are the equations (20), 21) and (23) in steady state $(k \rightarrow \infty)$. The Riccati equations are

$$
\begin{aligned}
& K_{1}=\frac{P_{11}^{\prime}}{P_{11}^{\prime}+\sigma_{w}^{2}} \\
& K_{2}=\frac{P_{21}^{\prime}}{P_{11}^{\prime}+\sigma_{w}^{2}} \\
& {\left[\begin{array}{ll}
P_{11}^{\prime} & P_{12}^{\prime} \\
P_{21}^{\prime} & P_{22}^{\prime}
\end{array}\right]=} \\
& {\left[\begin{array}{cc}
a_{1}^{2} P_{11}+a_{1} a_{2} P_{12}+a_{1} a_{2} P_{21}+a_{2}^{2} P_{22}+\sigma_{u}^{2} & a_{1} P_{11}+a_{2} P_{21} \\
a_{1} P_{11}+a_{2} P_{12} & P_{11}
\end{array}\right]} \\
& {\left[\begin{array}{ll}
P_{11} & P_{12} \\
P_{21} & P_{22}
\end{array}\right]=} \\
& {\left[\begin{array}{cc}
\left(1-K_{1}\right) P_{11}^{\prime} & \left(1-K_{1}\right) P_{12}^{\prime} \\
\left(-K_{2}\right) P_{11}^{\prime}+P_{21}^{\prime} & \left(-K_{2}\right) P_{21}^{\prime}+P_{22}^{\prime}
\end{array}\right] \text {. }}
\end{aligned}
$$


It can be seen from 24 that we need to find $P_{11}^{\prime}$ in order to deduce $K_{1}$. An exact formula for $P_{11}^{\prime}$ is given in Appendix B.1 and for $K_{2}$ in Appendix B.2.

\subsection{An approximate expression for $K_{1}$}

The expression for $K_{1}$ established previously is not mathematically tractable enough to get an analytical expression for the optimization of $\sigma_{\epsilon}^{2}$. To do so, the exact expression has to be approximated in closed form, by using the following assumptions.

\subsubsection{Assumptions used for the approximation}

(i) $f_{d} T \ll 1$ and then $f_{\mathrm{AR}(2)} T \ll 1$, i.e. slow fading assumptions.

(ii) $\sigma_{w} \leq \sigma_{\alpha}$, i.e. the SNR is greater than or equal to 1 .

(iii) $\delta \ll 1$ and (iv) $\zeta_{\operatorname{AR}(2)} \ll 1$, i.e. the presence of a pair of even symmetric peaks in the spectrum.

(v) $\sigma_{u} \ll \sigma_{w}$, i.e. the process noise is small compared to the measurement noise, as is the case in most applications [44, 7] with usual SNR (ii).

(vi) $\left(2 \pi f_{\mathrm{AR}(2)} T\right)^{2} \ll \frac{\sigma_{u}}{\sigma_{w}}$ it will be seen afterwards, at the end of this section (through (29), also (34)), that this means that the Kalman filter is sufficiently fast compared to the fading process.

Assumptions [i), (ii), (iii) and (iv) lead to the following relation (see Appendix A.3):

(vii) $\delta^{2} \ll \frac{\sigma_{u}}{\sigma_{w}}$. 


\subsubsection{Expression for $\sigma_{u}^{2}$}

Using Assumptions (i) and (iii), an expression for $\sigma_{u}^{2}$ is developed in terms of $r$ and $f_{\mathrm{AR}(2)} T$ in Appendix $\mathrm{H}$. The approximate expression for $\sigma_{u}^{2}$ is

$$
\sigma_{u}^{2} \simeq 4 \sigma_{\alpha}^{2} r(1-r)\left(\omega_{\mathrm{AR}(2)} T\right)^{2}
$$

where $\omega_{\mathrm{AR}(2)} T=2 \pi f_{\mathrm{AR}(2)} T$.

It should be noted that $r$ is the only variable in (28), due to the fact that $\omega_{\mathrm{AR}(2)} T$ is fixed using (12). For the purpose of simplicity, the optimization will be carried out with respect to $\sigma_{u}^{2}$ (or a parameter directly linked to $\sigma_{u}^{2}$ ) instead of $r$, and the results will then be expressed in terms of $r$ by using 28).

\subsubsection{Formula for $K_{1}$}

Based on Assumptions (i) (v), we prove in Appendix C that the exact expression for $K_{1}(24)$ can be approximated by (29), and then by (30) in using (28):

$$
\begin{aligned}
K_{1} & \simeq \sqrt{\frac{2 \sigma_{u}}{\sigma_{w}}} \\
& \simeq \sqrt{\frac{4 \sigma_{\alpha} r^{\frac{1}{2}}(1-r)^{\frac{1}{2}} \omega_{\mathrm{AR}(2)} T}{\sigma_{w}}} .
\end{aligned}
$$

From (29) and Assumption (v) it can be deduced that

(viii) $K_{1} \ll 1$ and from (vi) and (vii) it can be respectively deduced that:

(ix) $K_{1} \gg \delta \quad$ and $\quad(\mathrm{x}) K_{1} \gg f_{\mathrm{AR}(2)} T$.

All these assumptions on $K_{1}$ mean that the Kalman gain has been chosen to be small due to (viii) because of the slow fading assumption but large enough 
due to (x) to perform the tracking (see (31) and (34), where $K_{1}$ plays the role of the normalized equivalent bandwidth). Equation (29) means that for a given noise level, the value of the Kalman gain $K_{1}$ directly increases with $\sigma_{u}$.

\section{Error Variance in Steady-State and Optimization}

Now that a closed-form expression for the Kalman gain has been established, we can proceed to the criterion. To do so, we first have to derive an expression for the error variance, i.e. the steady state MSE, as a function of the Kalman gain in 4.1. Then, the optimization is carried out in 4.2 .

\subsection{Error variance in steady-state}

Write $\hat{\alpha}(z), \alpha(z), y(z), w(z)$ for the $z$-transforms of $\hat{\alpha}_{(k \mid k)}, \alpha_{(k)}, y_{(k)}$ and $w_{(k)}$, respectively. Denote by $L(z)$ the transfer function of the filter that gives $\hat{\alpha}(z)$ with the observation $y(z)$ as input. In a steady state, the solution is given by the system of equations (19) and (22) of the KF by (see Appendix E)

$$
L(z)=\frac{K_{1}+a_{2} K_{2} z^{-1}}{1+z^{-1}\left(a_{2} K_{2}-a_{1}\left(1-K_{1}\right)\right)-a_{2}\left(1-K_{1}\right) z^{-2}},
$$

where $a_{1}$ and $a_{2}$ were defined in (11) in terms of $r$ (and then $K_{1}$ from (30) and $K_{2}$ is expressed in $(\mathrm{B} .20)$ in terms of $a_{1}, a_{2}$ and $K_{1}$. In comparison with second order systems [34], this corresponds to a second-order low-pass fil-

ter with normalized natural frequency $\omega_{n, L(z)} T \simeq \frac{K_{1}}{\sqrt{2}}$ and a damping factor $\zeta_{L(z)} \simeq \frac{\sqrt{2}}{2}$ Appendix F.

From the filtering equations, we have $\hat{\alpha}(z)=L(z)(\alpha(z)+w(z))$ (illustrated 


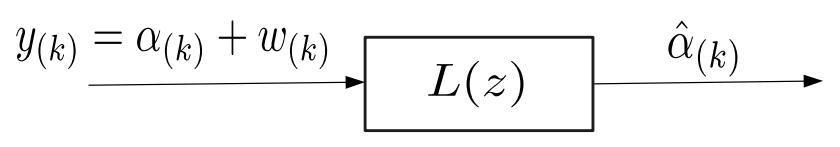

Figure 4: Scheme of the KF in steady-state

in Fig. (4) and the estimation error $\epsilon(z)$ is

$$
\epsilon(z)=\alpha(z)-\hat{\alpha}(z)=(1-L(z)) \alpha(z)-L(z) w(z) .
$$

Therefore, it remains to calculate the power of the error from $\epsilon(z)$ 32], which can be split into two additive contributions:

$$
\sigma_{\epsilon}^{2}=\sigma_{\epsilon w}^{2}+\sigma_{\epsilon \alpha}^{2}
$$

- The static error variance $\sigma_{\epsilon w}^{2}$ is due to the additive noise $w_{(k)}$ filtered by the low pass filter $L(z)$. The details of the calculation, together with the analytical results, are given in Appendix G, where we also derive an approximate expression by resorting to Assumptions (i), (ii), (iii), (iv), (v), and (viii)

$$
\sigma_{\epsilon w}^{2} \simeq \sigma_{w}^{2} \frac{3 K_{1}}{4} .
$$

Equation 34 means that $\frac{3 K_{1}}{4}$ plays the role of the normalized equivalent bandwidth of the closed-loop steady-state Kalman $L(z)$, which a posteriori sheds light on Assumptions (v), (vi), (viii) and (x),

- The dynamic error variance $\sigma_{\epsilon \alpha}^{2}$ is due to the variations of $\alpha_{(k)}$ filtered by the high pass filter $1-L(z)$ :

$$
\sigma_{\epsilon \alpha}^{2} \simeq \sigma_{\alpha}^{2} \frac{6 \pi^{4}\left(f_{d} T\right)^{4}}{K_{1}^{4}}
$$


An exact expression for $\sigma_{\epsilon \alpha}^{2}$ as well as an approximation for it are given in Appendix G.1.

In summary, we have the global MSE $\sigma_{\epsilon}^{2}$ in terms of $K_{1}$ and $f_{d} T$ :

$$
\sigma_{\epsilon}^{2} \simeq \sigma_{w}^{2} \frac{3 K_{1}}{4}+\sigma_{\alpha}^{2} \frac{6 \pi^{4}\left(f_{d} T\right)^{4}}{K_{1}^{4}}
$$

where $K_{1}$ is in terms of $\sigma_{u}$ and $\sigma_{w}$ (see $(29)$ ).

\subsection{Optimization}

In this section, we first look for the minimization of $\sigma_{\epsilon}^{2}$. To do so, we proceeded in two steps. First, we find the optimal $\sigma_{u}^{2}$, denoted by $\sigma_{u(\mathrm{MAV})}^{2}$, that minimizes $\sigma_{\epsilon}^{2}$. Then, the expressions for $r$ and $\zeta_{\mathrm{AR}(2)}$ are deduced from $\sigma_{u(\mathrm{MAV})}^{2}$ in terms of the channel state $\left(f_{d} T\right.$ and SNR).

Thus, expressing (36) with 29 , differentiating it with respect to $\sigma_{u}^{2}$, and equating the derivative to zero, yields

$$
\sigma_{u(\mathrm{MAV})}^{2}=4 \pi^{\frac{16}{5}}\left(\sigma_{\alpha}^{2}\left(f_{d} T\right)^{4} \sqrt{\sigma_{w}}\right)^{\frac{4}{5}}
$$

and the theoretical minimum MSE

$$
\sigma_{\epsilon(\operatorname{AR}(2)-\mathrm{MAV})}^{2}=\frac{15}{8} \pi^{\frac{4}{5}}\left(\sigma_{\alpha}^{2}\right)^{\frac{1}{5}}\left(f_{d} T \sigma_{w}^{2}\right)^{\frac{4}{5}}
$$

Part of this work has been presented in [1], but without rigorous justifica-

tions. In [1], a sub-optimal adjustment of $\left\{a_{1}, a_{2}\right\}$ was proposed by giving an analytical expression for $a_{1}$ in terms of $a_{2}$, where $a_{2}$ is given by imposing a linear constraint obtained through experimentation, with respect to the Doppler frequency. In the following, the optimal tuning of $r$ and $\zeta_{\mathrm{AR}(2)}$ is given in terms of the Doppler frequency and the SNR. This is one of the contributions of the present paper. 


\subsubsection{Optimal tuning of $r$ and $\zeta_{A R(2)}$}

The optimal tuning of the parameters $r$ and $\zeta_{\operatorname{AR}(2)}$ are given in this section. Using the expression for $\sigma_{u}^{2}$ defined in 28,

$$
\frac{\sigma_{u}^{2}}{4 \sigma_{\alpha}^{2}\left(\omega_{\mathrm{AR}(2)} T\right)^{2}} \simeq(1-r) r .
$$

Hence $r$ is one solution of the second degree equation

$$
r^{2}-r+\frac{\sigma_{u}^{2}}{4 \sigma_{\alpha}^{2}\left(\omega_{\mathrm{AR}(2)} T\right)^{2}} \simeq 0 .
$$

Solving this equation, we find

$$
r \simeq \frac{1}{2}+\frac{1}{2} \sqrt{1-\frac{\sigma_{u}^{2}}{\sigma_{\alpha}^{2}\left(\omega_{\mathrm{AR}(2)} T\right)^{2}}}
$$

Due to the fact that $\frac{\sigma_{u}^{2}}{\sigma_{\alpha}^{2}\left(\omega_{\mathrm{AR}(2)} T\right)^{2}} \simeq 4(1-r) r \ll 1$ (Eq. 39p), we have

$$
r \simeq 1-\frac{\sigma_{u}^{2}}{4 \sigma_{\alpha}^{2}\left(\omega_{\mathrm{AR}(2)} T\right)^{2}} .
$$

Equation (42) is always valid under the assumptions 3.3.1.

In the special case of the MAV criterion, one can calculate the optimal radius $r_{\text {(MAV) }}$ from the optimal state noise $\sigma_{u(\mathrm{MAV})}^{2}(37)$ :

$$
\begin{aligned}
r_{(\mathrm{MAV})} & =1-\frac{\sigma_{u(\mathrm{MAV})}^{2}}{4 \sigma_{\alpha}^{2}\left(\omega_{\mathrm{AR}(2)} T\right)^{2}} \\
& =1-\frac{\pi^{\frac{6}{5}}\left(f_{d} T\right)^{\frac{6}{5}}\left(\frac{\sigma_{w}^{2}}{\sigma_{\alpha}^{2}}\right)^{\frac{1}{5}}}{2},
\end{aligned}
$$

where $\omega_{\mathrm{AR}(2)} T=2 \pi f_{\mathrm{AR}(2)} T$ and $f_{\mathrm{AR}(2)} T$ is fixed using the optimal choice already defined in (12). Having $r_{(\mathrm{MAV})}$, an expression for the dumping ratio of 
the $\mathrm{AR}(2)$ process $\zeta_{\mathrm{AR}(2)}$ (MAV) can be deduced from $(15)$, using that $r=1-\delta$ :

$$
\zeta_{\mathrm{AR}(2)(\mathrm{MAV})} \simeq \frac{1-r_{(\mathrm{MAV})}}{\omega_{\mathrm{AR}(2)}} \simeq \frac{\sqrt{2}}{4}\left(\pi f_{d} T \frac{\sigma_{w}^{2}}{\sigma_{\alpha}^{2}}\right)^{\frac{1}{5}}
$$

The above equation gives insight into the shape of the PSD of the optimal $\mathrm{AR}(2)$ process. As mentioned previously, the height of the peak is inversely proportional to $\zeta_{\mathrm{AR}(2)}$. Thus, (44) means that the greater the SNR and the lower the $f_{d} T$, the higher the peak. This will be illustrated in the simulation section.

\subsection{Comparison with $A R(1)-M A V$ and $R W(2)$}

The AR(1)-MAV has been obtained in [28], where the theoretical MSE was calculated as follows:

$$
\sigma_{\epsilon(\operatorname{AR}(1)-\mathrm{MAV})}^{2} \simeq \frac{3}{2} \cdot \pi^{\frac{2}{3}}\left(\sigma_{\alpha}^{2}\right)^{\frac{1}{3}}\left(f_{d} T \sigma_{w}^{2}\right)^{\frac{2}{3}}
$$

Comparing (38) and (45), we have

$$
\sigma_{\epsilon(\operatorname{AR}(2)-\mathrm{MAV})}^{2}<\sigma_{\epsilon(\mathrm{AR}(1)-\mathrm{MAV})}^{2}
$$

It will be seen later, in the simulation section, that $\mathrm{AR}(2)-\mathrm{MAV}$ outperforms AR(1)-MAV, which confirms the comparison above.

On the other hand, the performance of the stationary $\mathrm{AR}(2)$ model under the MAV criterion is close to the one obtained with a non-stationary model, the second order random walk $(\mathrm{RW}(2))$ model [33] under the same criterion:

$$
\frac{\sigma_{\epsilon(\mathrm{RW}(2)-\mathrm{MAV})}^{2}}{\sigma_{\epsilon(\mathrm{AR}(2)-\mathrm{MAV})}^{2}}=2^{\frac{2}{5}}
$$




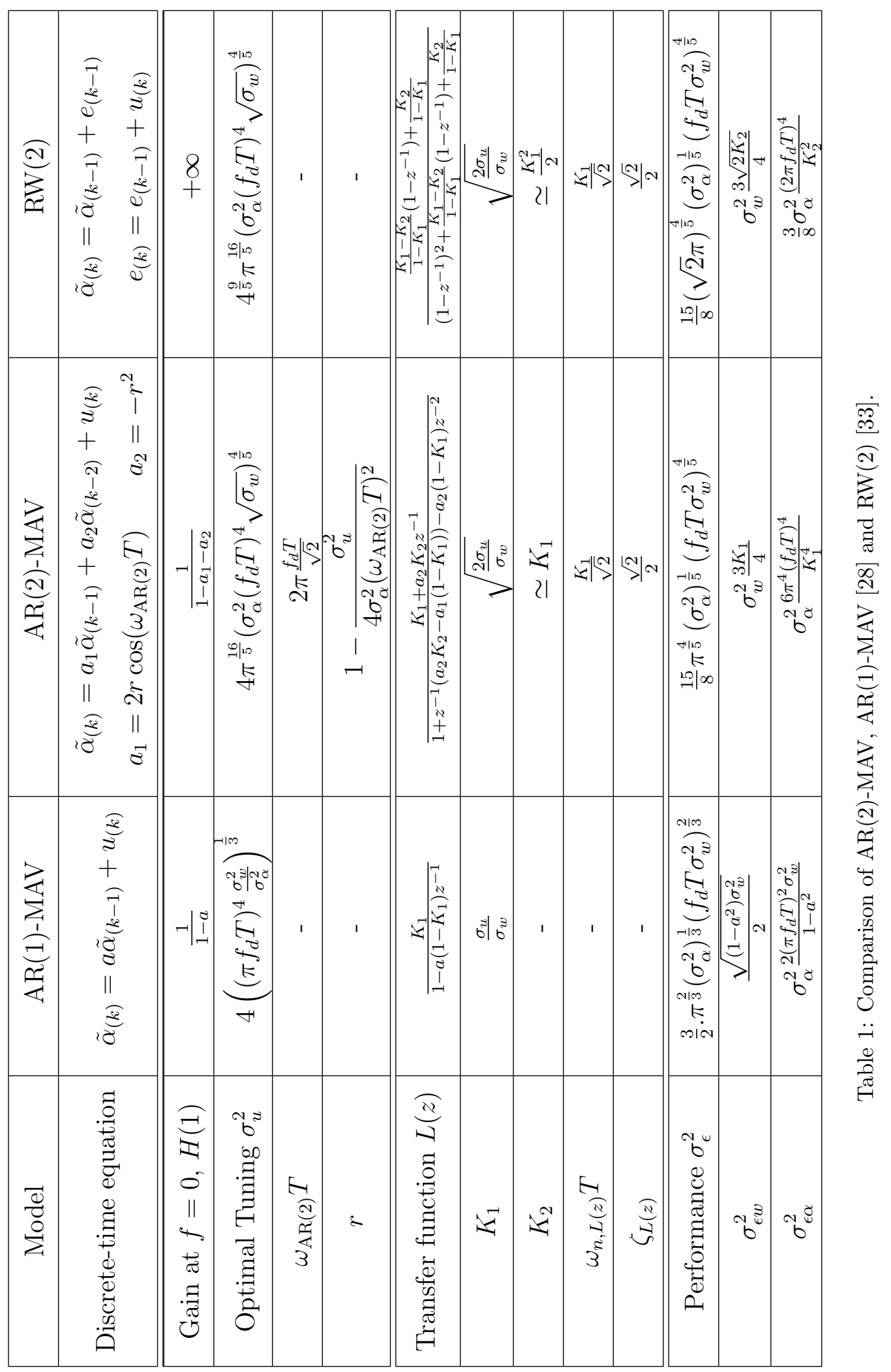


Note that the expression for the transfer function $L(z)$ obtained in (31) for the $\mathrm{AR}(2)-\mathrm{KF}$ is different from the one obtained in [33] for the RW(2) model, due to the difference between the two discrete-time second-order systems. In addition, the two components of the Kalman gain are nearly equal ( $K_{2} \simeq K_{1}$, see Appendix D for the AR(2) model, while this is not the case for the RW(2) model, for which $K_{2} \simeq \frac{K_{1}^{2}}{2}$ 33]. Despite these differences, the damping factors (issuing from the equivalent approximate analog second-order systems) of the two KFs have the same value $\zeta_{L(z)} \approx \frac{\sqrt{2}}{2}$, which means that the shapes of the frequency domain transfer functions $L\left(e^{j 2 \pi f T}\right)$ of the $\mathrm{AR}(2)-\mathrm{KF}$ and RW(2)-KF are very close, under the slow fading assumption. Also, in both cases, the normalized natural frequency is linked to the first component of the Kalman gain by $\omega_{n, L(z)} T \approx K_{1} / \sqrt{2}$. Table 1 provides a clear presentation of the differences and common points between the AR(2)-MAV, AR(1)MAV 28] and RW(2) 33] Kalman filters.

\section{Simulation Results}

\subsection{Illustration of system parameters and interpretation}

The objective of this section is to illustrate and interpret the values of the

optimal system parameters for a given channel state $\left(f_{d} T\right.$ and SNR), and to compare them with other possible choices in the literature. The autocorrelation functions (ACFs) as well as the PSDs of the $\operatorname{AR}(2)$ processes are discussed and illustrated. A comparison of the MSEs obtained by Monte Carlo simulations for different values of $f_{\mathrm{AR}(2)} T$ is first presented in Fig. 5 , to assess the value adopted from the literature and the significance of the 
MAV criterion.

\subsubsection{MSE for different values of $f_{A R(2)} T$}

Figure 5 shows the impact of the parameter $f_{\mathrm{AR}(2)} T$ on the asymptotic MSE. In order to illustrate the choice $f_{\mathrm{AR}(2)} T$ in $(12)$, Monte Carlo simulations have been carried out for $f_{\mathrm{AR}(2)} T=\left\{\frac{f_{d} T}{2}, \frac{f_{d} T}{\sqrt{2}}, f_{d} T\right\}$ at different SNR and $f_{d} T$. They confirm [31] and [30], claiming that $f_{\mathrm{AR}(2)} T=\frac{f_{d} T}{\sqrt{2}}$ is the optimal choice in terms of MSE. Note also that the AR(2)-CM performs poorly compared to $\operatorname{AR}(2)-\mathrm{MAV}$ with $f_{\mathrm{AR}(2)} T=\frac{f_{d} T}{\sqrt{2}}$.

\subsection{2. $A C F$ of $A R(2)$}

In this subsection, the Yule-Walker [14] equations are used to get the ACF of the $\operatorname{AR}(2)$ :

$$
R_{\tilde{\alpha}}[k]=a_{1} R_{\tilde{\alpha}}[k-1]+a_{2} R_{\tilde{\alpha}}[k-2] .
$$

Figure 6 plots the ACFs of different AR(2) processes: the $\mathrm{AR}(2)-\mathrm{MAV}$ with $f_{\mathrm{AR}(2)} T=\frac{f_{d} T}{\sqrt{2}}$ and $f_{\mathrm{AR}(2)} T=f_{d} T$, and the $\mathrm{AR}(2)$-CM. For comparison, the true ACF, i.e. the Bessel function (3), is also plotted. As expected from our discussions in Section 2, it can be seen that the ACF of the AR(2)-MAV with $f_{\mathrm{AR}(2)} T=\frac{f_{d} T}{\sqrt{2}}$ is very close (in terms of Euclidean distance) to the true ACF (Bessel function), for the first lobe region.

\subsubsection{PSD of optimal $A R(2)$}

Figure 7 shows the PSDs of the optimal AR(2)-MAV with $f_{\operatorname{AR}(2)} T=\frac{f_{d} T}{\sqrt{2}}$ for different values of SNR and $f_{d} T$. It can be seen that the level of the peaks depends on the SNR and $f_{d} T$. More precisely, the highest value of the peak 
is obtained for the lowest $f_{d} T=10^{-4}$ and the highest $\mathrm{SNR}=20 \mathrm{~dB}$, which is consistent with (44). Moreover, it is interesting to compare these levels with the one obtained with the AR(2)-CM. In fact, the example of the PSD given in Fig. 3 has been obtained with the CM criterion $\left(\delta=2.46 \times 10^{-6}\right.$ in Fig. 3). The PSD for the CM has an overhead of about $40 \mathrm{~dB}$ compared to the value at $0 \mathrm{~Hz}$, and this is independent of the SNR. Indeed, the CM solution for $a_{1}$ and $a_{2}$ does not depend on the SNR. This value, $40 \mathrm{~dB}$, is to be compared with the one obtained for the MAV, which is only about $12 \mathrm{~dB}$ for $\mathrm{SNR}=0 \mathrm{~dB}$ and $f_{d} T=10^{-3}$.

\subsubsection{Dependence of the optimal parameters on the channel state}

In this subsection, the dependence of $\zeta_{\mathrm{AR}(2)(\mathrm{MAV})}$ and $\delta_{(\mathrm{MAV})}=1-r_{(\mathrm{MAV})}$ on the channel state $\left(f_{d} T\right.$ and SNR) is discussed. Both parameters are plotted as functions of $f_{d} T$ for the three values of $\mathrm{SNR}=0,20,40 \mathrm{~dB}$ in Fig. 11. It can be seen that $\delta_{(\mathrm{MAV})}=1-r_{(\mathrm{MAV})}$ increases proportionally to the $\frac{6}{5}$ th power of $f_{d} T$, and decreases proportionally to the $\frac{1}{5}$ th power of the SNR, which agrees with 43 . In the same way, we can see that $\zeta_{\mathrm{AR}(2) \text { (MAV) increases }}$ proportionally to the $\frac{1}{5}$ th power of the product $\left(\frac{f_{d} T}{\mathrm{SNR}}\right)$, which is in accordance with (44). As was mentioned in the previous section, the power spectral density depends on $\zeta_{\mathrm{AR}(2)(\mathrm{MAV})}$, in that $\zeta_{\mathrm{AR}(2)(\mathrm{MAV})}$ influences the overhead of the peak of $H(z)$. The maximum value of $\zeta_{\mathrm{AR}(2)(\mathrm{MAV})}$, about 0.1 , is obtained for the minimum $\mathrm{SNR}=0 \mathrm{~dB}$ and maximum $f_{d} T=10^{-2}$, which again illustrates the fact that the peak is the lowest at the minimum SNR and maximum $f_{d} T$. Even in this worst case, the second-order model is still underdamped. 


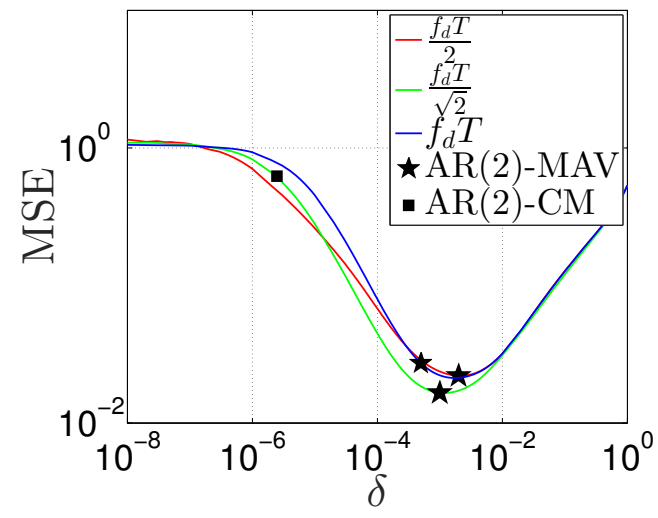

(a) $f_{d} T=10^{-3}-\mathrm{SNR}=0 \mathrm{~dB}$

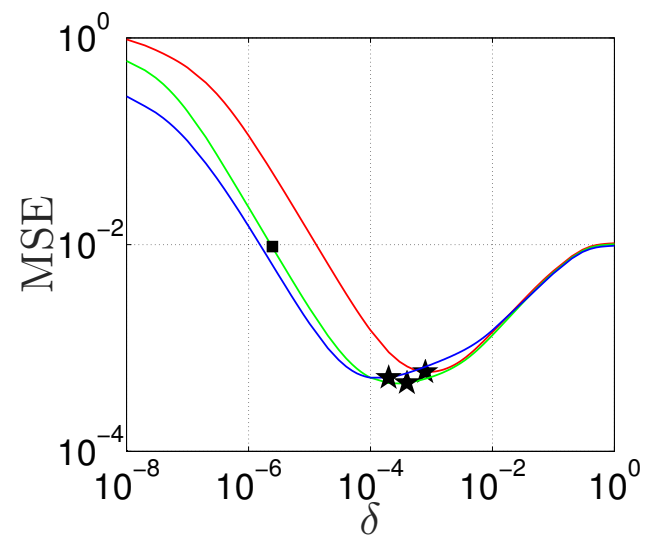

(c) $f_{d} T=10^{-3}-\mathrm{SNR}=20 \mathrm{~dB}$

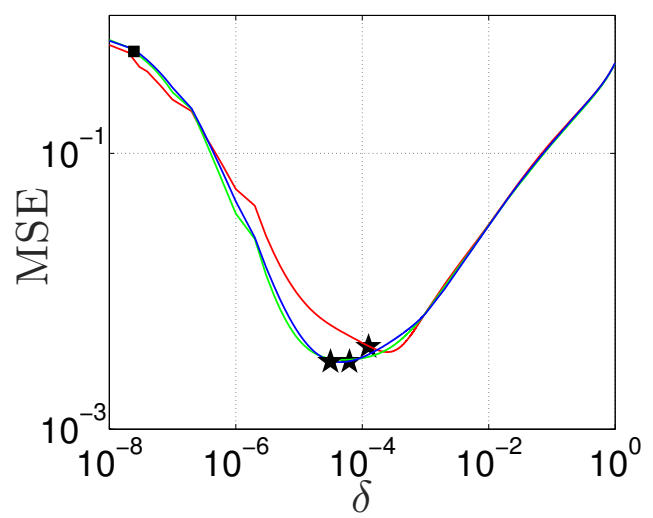

(b) $f_{d} T=10^{-4}-\mathrm{SNR}=0 \mathrm{~dB}$

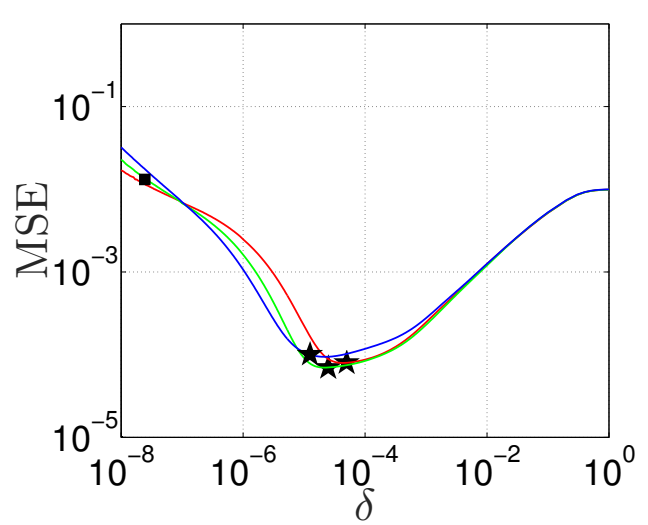

(d) $f_{d} T=10^{-4}-\mathrm{SNR}=20 \mathrm{~dB}$

Figure 5: Variation of MSE versus $\delta=1-r$ for different values of $f_{\mathrm{AR}(2)} T$ for $\mathrm{SNR}=0$ $\mathrm{dB}$ and $20 \mathrm{~dB}$. 


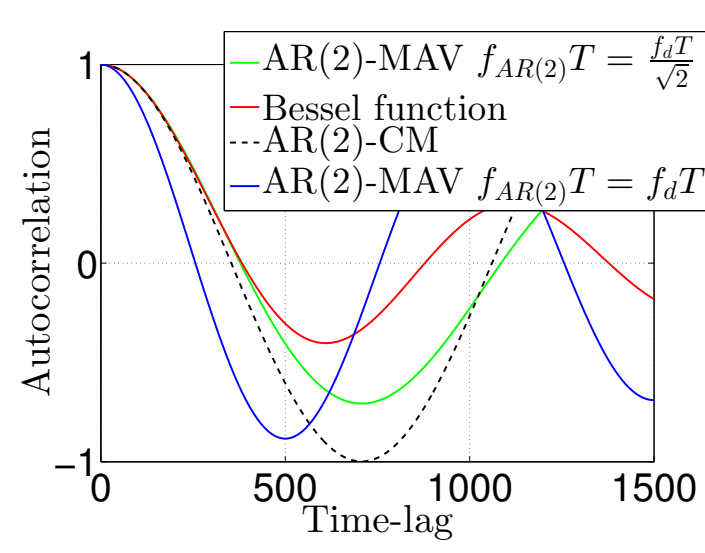

(a) $f_{d} T=10^{-3}-\mathrm{SNR}=0 \mathrm{~dB}$

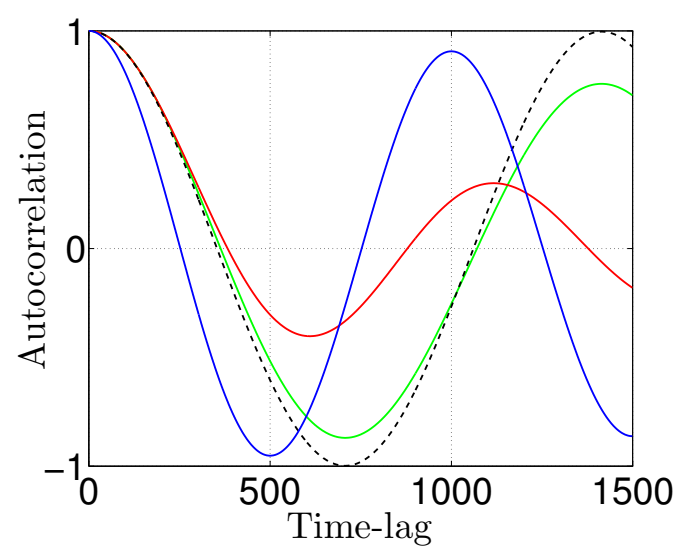

(c) $f_{d} T=10^{-3}-\mathrm{SNR}=20 \mathrm{~dB}$

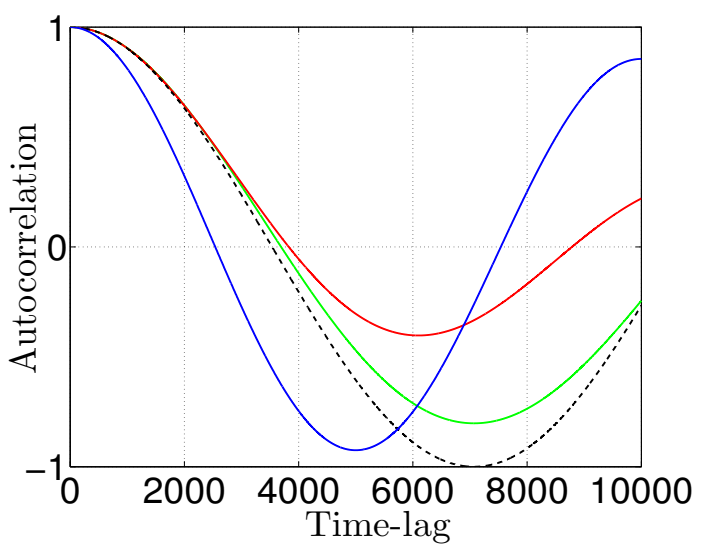

(b) $f_{d} T=10^{-4}-\mathrm{SNR}=0 \mathrm{~dB}$

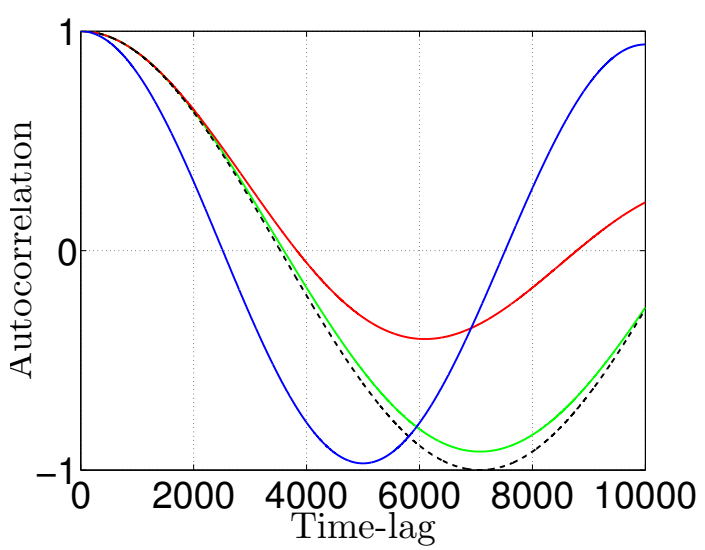

(d) $f_{d} T=10^{-4}-\mathrm{SNR}=20 \mathrm{~dB}$

Figure 6: Autocorrelation functions of $\mathrm{AR}(2)$ with $\mathrm{CM}$ and MAV criteria and Bessel function for $f_{d} T=10^{-3}$ and $f_{d} T=10^{-4}$ for SNR $=0 \mathrm{~dB}$ and $20 \mathrm{~dB}$. 


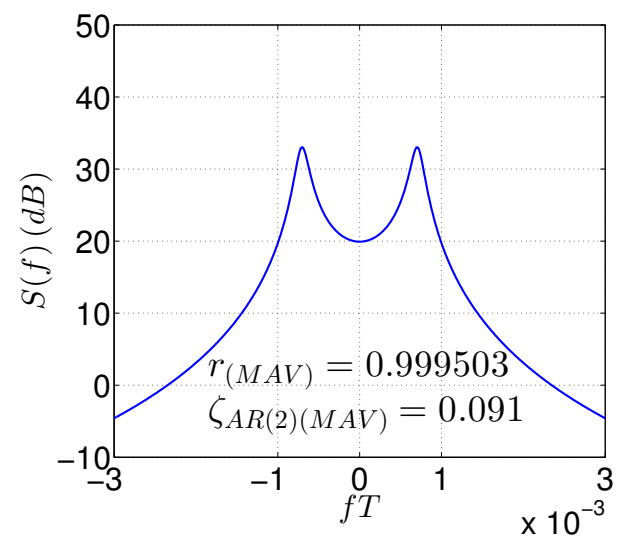

(a) $f_{d} T=10^{-3}, \mathrm{SNR}=0 \mathrm{~dB}$

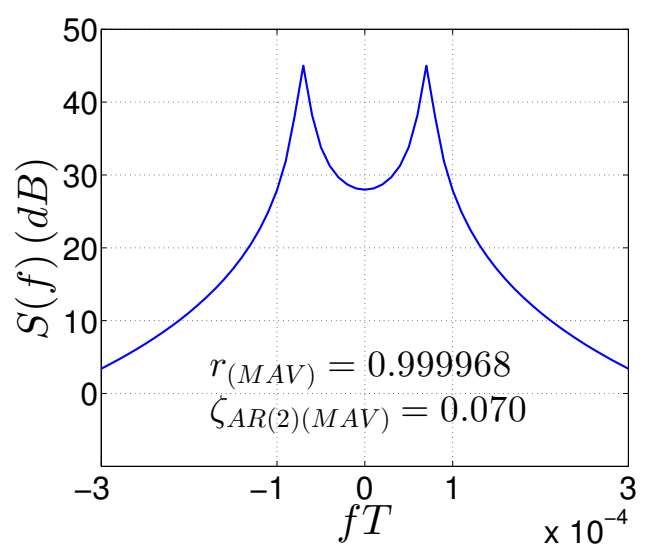

(c) $f_{d} T=10^{-4}, \mathrm{SNR}=0 \mathrm{~dB}$

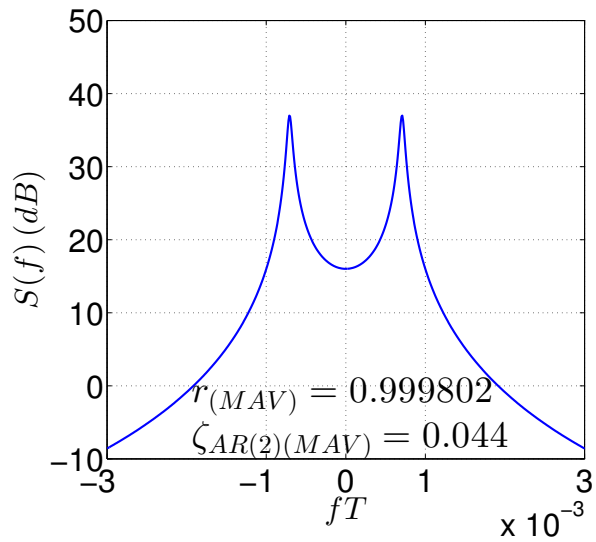

(b) $f_{d} T=10^{-3}, \mathrm{SNR}=20 \mathrm{~dB}$

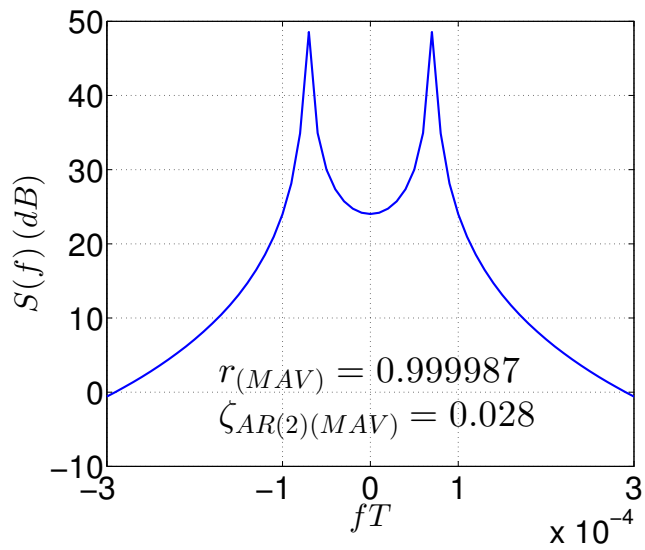

(d) $f_{d} T=10^{-4}, \mathrm{SNR}=20 \mathrm{~dB}$

Figure 7: Spectrum of $\mathrm{AR}(2)$ with MAV criterion for various $f_{d} T$ and SNR. 


\subsection{Performance}

\subsubsection{Performance in terms of $M S E$}

Figure 8 plots the asymptotic MSE of the KF estimates when the $\operatorname{AR}(2)$ model is tuned with our proposed MAV criterion (AR(2)-MAV). For comparison, the $\mathrm{KF}$ with the following $\mathrm{AR}(2)$ models from the literature are considered: the $\mathrm{AR}(2)-\mathrm{CM}$ [11 13] together with the improved $\mathrm{AR}(2)-\mathrm{CM}$ with $\epsilon$ [9, the $\operatorname{AR}(2)$ of [4, 31] where $r$ is tuned based on experimental results as follows: $r=0.999-0.1 \times 2 \pi f_{d} T$ and $f_{\mathrm{AR}(2)} T=\frac{f_{d} T}{\sqrt{2}}$. Note that the expression for $r$ does not depend on the SNR.

Moreover, due to the fact that the least mean square (LMS) algorithm (or integrated versions) gives similar steady-space performance as some KF (with random-walk state-space models [40] [41]), the AR(2)-MAV KF is compared to a set of LMS adaptive algorithms:

1. The Wiener least mean square algorithm (WLMS) proposed by [40]. In this model, the $\mathrm{AR}(2)$ process is used for the prediction of the next CG.

2. The adaptive LMS (A-LMS) algorithm by [41], where a second order random walk model is used.

The on-line BCRB [45] is also plotted.

First, it can be verified that the MSE computed by simulation is very close to $\sigma_{\epsilon(\operatorname{AR}(2)-\mathrm{MAV})}^{2}$ as obtained by the closed-form expression in 38 , thus validating the theoretical analysis. Then, it is seen in Fig. 8 that the AR(2)-MAV outperforms the other algorithms from the literature. Fig. 9 plots the MSE of the WLMS, A-LMS, AR(2) [13] and AR(2)-MAV as a function of $f_{d} T$ 


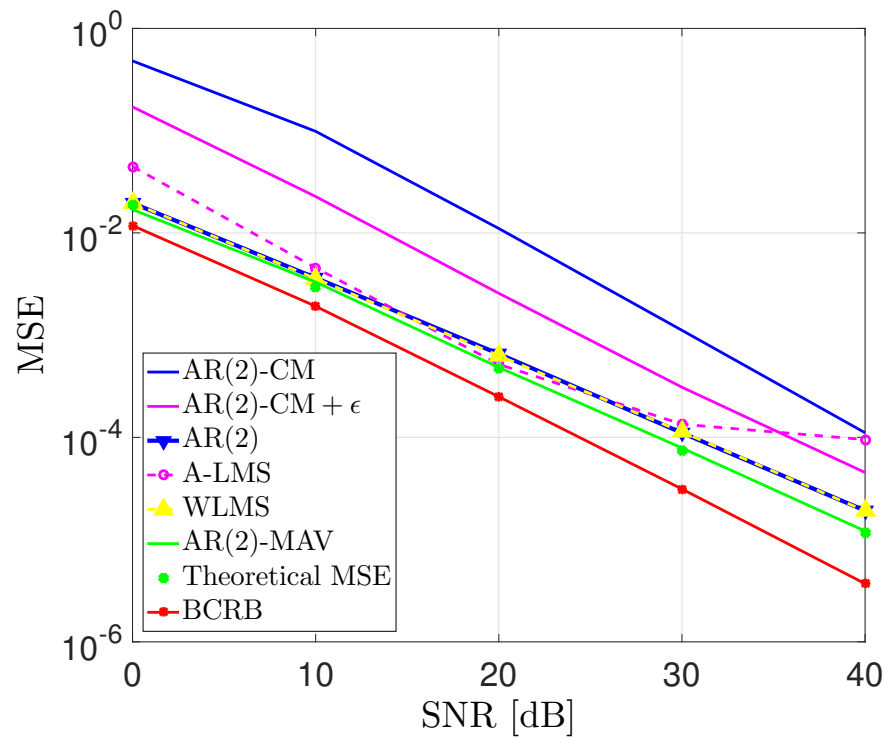

(a) $f_{d} T=10^{-3}$

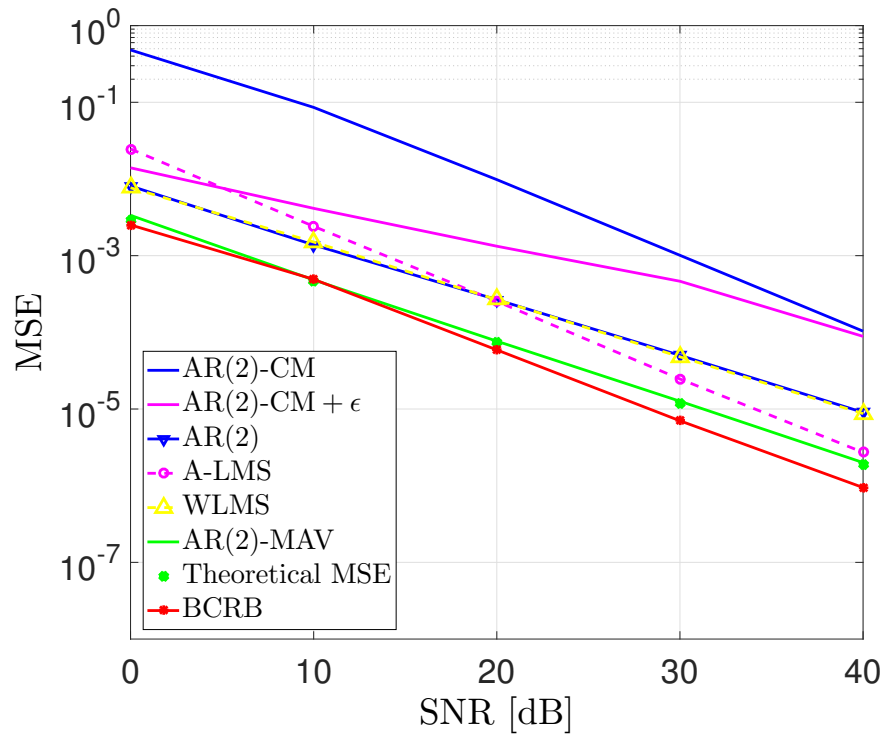

(b) $f_{d} T=10^{-4}$

Figure 8: Comparison of the asymptotic MSE of the Kalman filters for proposed AR(2)MAV with the literature: $\mathrm{AR}(2)-\mathrm{CM}$ [11 13, $\mathrm{AR}(2)-\mathrm{CM}+\epsilon$ [9], $\mathrm{AR}(2)$ [4, A-LMS 41], WLMS [40, in terms of SNR for $f_{d} T=10^{-4}$ and $f_{d} T=10^{-3}$. 


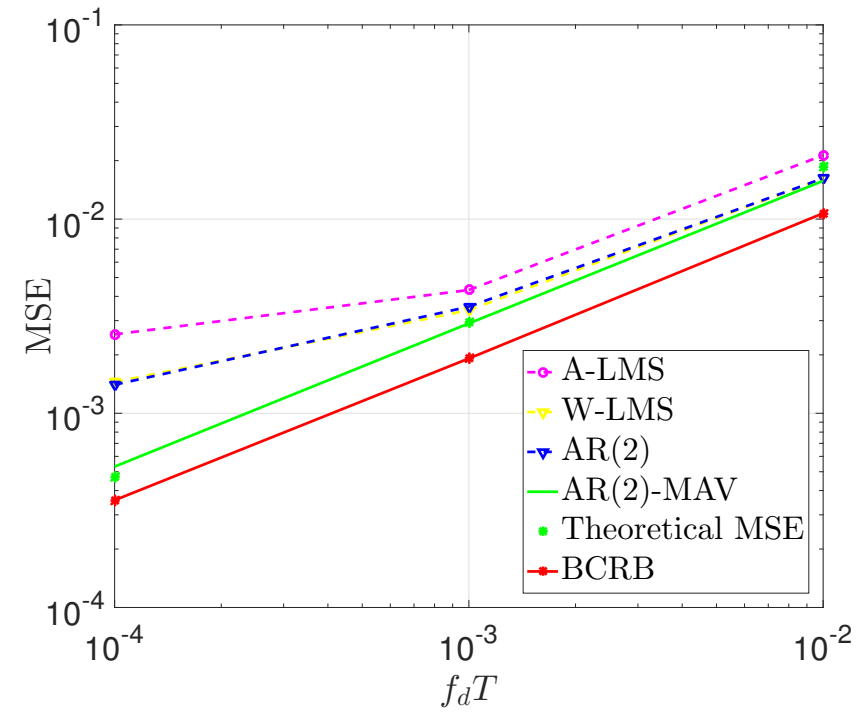

Figure 9: Comparison of the asymptotic MSE of the proposed AR(2)-MAV with the literature: A-LMS [1], W-LMS 40], $\operatorname{AR}(2)$ 4, for different values of $f_{d} T$ and SNR $=10$ dB.

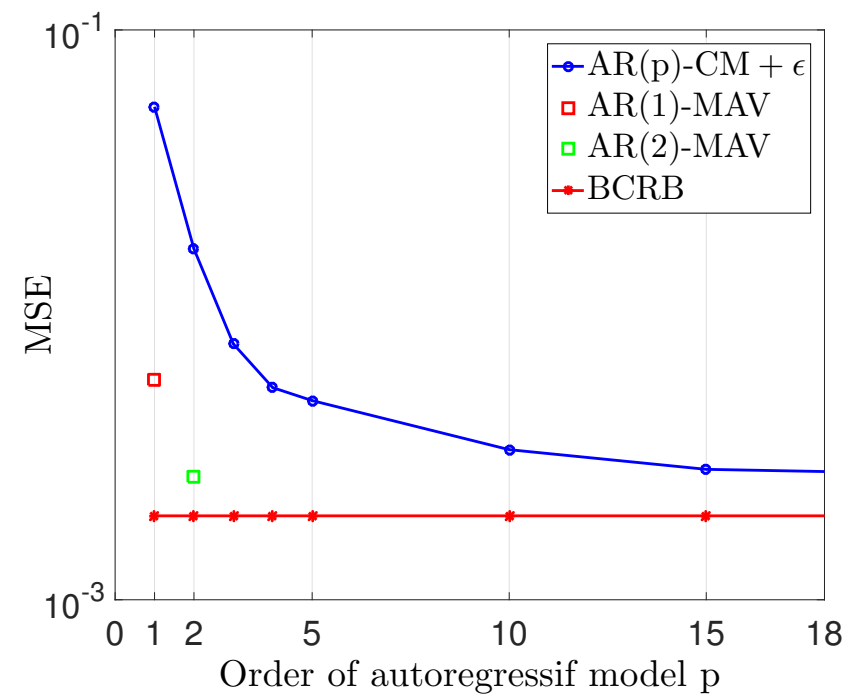

Figure 10: Comparison of $\mathrm{AR}(1)-\mathrm{MAV}$ 28, $\mathrm{AR}(2)-\mathrm{MAV}$ and $\mathrm{AR}(p)-\mathrm{CM}+\epsilon$ where $\epsilon$ is set according to 9 for $f_{d} T=10^{-3}$ and SNR $=10 \mathrm{~dB}$. 


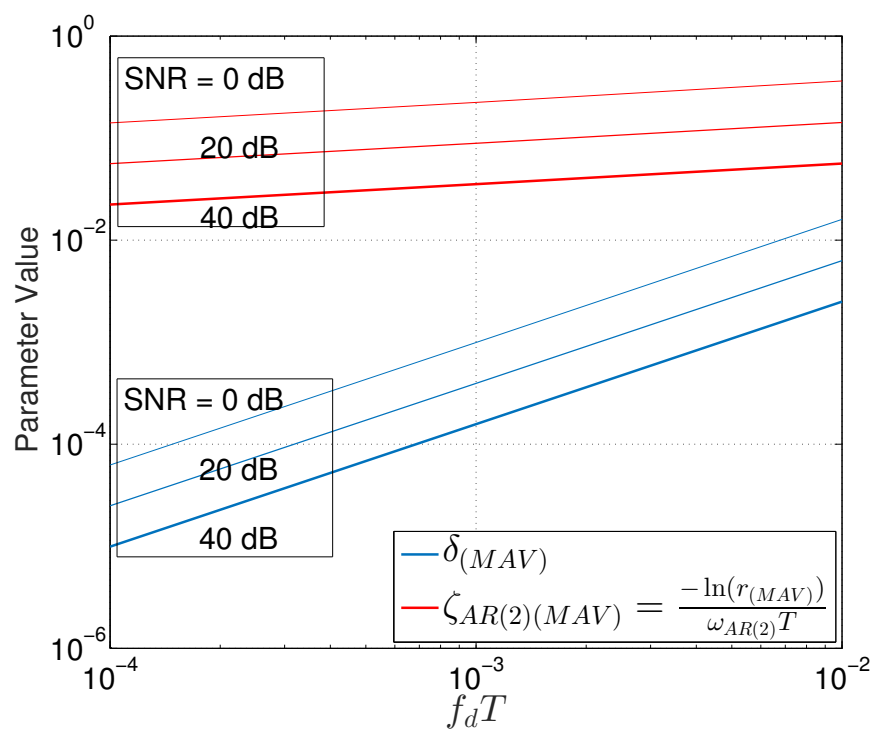

Figure 11: $\delta_{(\mathrm{MAV})}=1-r_{(\mathrm{MAV})}$ 43 and $\zeta_{\mathrm{AR}(2) \mathrm{MAV}} 44$ for different values of $f_{d} T$ and SNR.

for $\mathrm{SNR}=10 \mathrm{~dB}$. The MSE obtained with the $\mathrm{AR}(2)-\mathrm{MAV}$ remains close to the BCRB, which is not the case for the other algorithms, especially for $f_{d} T=10^{-4}$. This result shows that the $\mathrm{AR}(2)$-MAV can be used up to $f_{d} T=10^{-2}$, which corresponds to moderate normalized Doppler frequencies.

Fig. 10 plots the MSE of the KF estimates as a function of the order $p$ of the AR model for the CM criterion [9] and the MAV criterion. The figure shows that the $\mathrm{AR}(2)-\mathrm{MAV}$ outperforms the $\mathrm{AR}(1)-\mathrm{MAV}$ [28], which is confirmed by the theory in (46). It shows also that the MSE of the AR(2)-MAV is equivalent to the one of the far more complex AR(15)-CM, which highlights the interest of our study. 


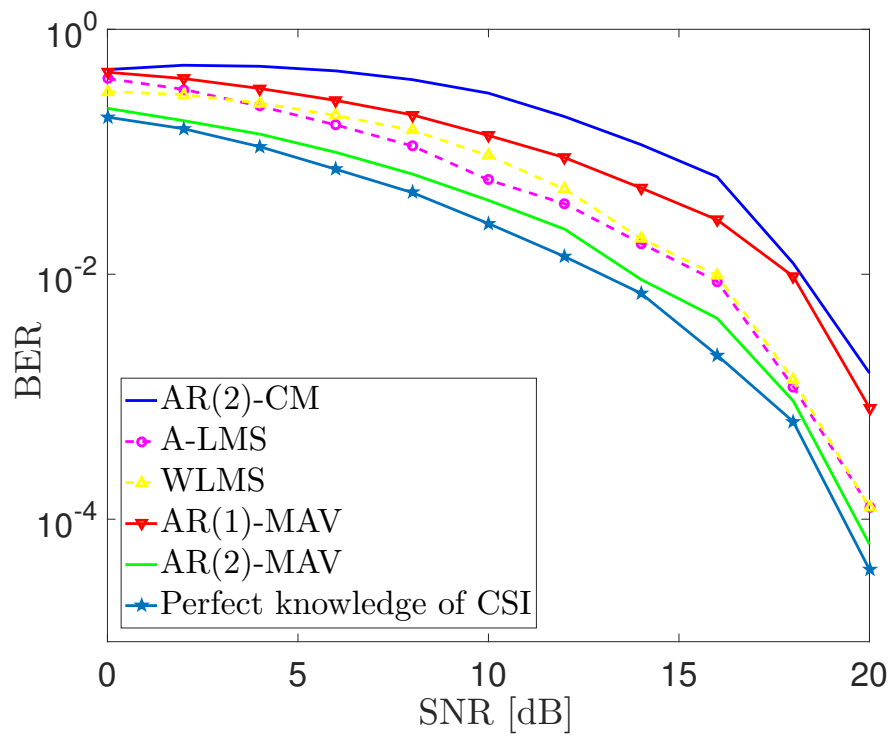

Figure 12: Comparison of the BER of the proposed AR(2)-MAV with the literature: AR(2)-CM[11 13], A-LMS 41, W-LMS[40, AR(1)-MAV 28], versus SNR for QPSK modulation and $f_{d} T=10^{-4}$.

\subsubsection{Performance in terms of BER}

Until now, all the performances were given in terms of the MSE. The performances in terms of the Bit Error Rate (BER) are given in Fig. 12, where QPSK transmitted symbols are used. The estimation is in semi-blind mode, that is, the data block is composed of 20 pilot symbols followed by 200 unknown symbols. The method used consists in changing the observation model in (1) to become $y_{(k)}=\alpha_{(k)} \times s_{(k)}+w_{(k)}$ where $s_{(k)}$ is a QPSK symbol. In this case, Eq. (22) of the KF equations is also modified by replacing $y_{(k)}$ by $y_{(k)} \times \hat{s}_{(k \mid k-1)}^{*}$, where $\hat{s}_{(k \mid k-1)}=s_{(k)}$ if $s_{(k)}$ is a pilot symbol or $\hat{s}_{(k \mid k-1)}=\operatorname{sgn}\left\{\left(\operatorname{Re}\left(y_{(k)} \times \hat{\alpha}_{(k \mid k-1)}^{*}\right)\right\}\right.$ if $s_{(k)}$ is an unknown symbol. In this case, $\hat{s}_{(k \mid k-1)}$ represents the decision a priori, and the final decision will be 
$\hat{s}_{(k \mid k)}=\operatorname{sgn}\left\{\left(\operatorname{Re}\left(y_{(k)} \times \hat{\alpha}_{(k \mid k)}^{*}\right)\right\}\right.$. Fig. 12 shows the simulation result, where it is seen that the AR(2)-MAV outperforms the literature, with a BER close to that with a perfect knowledge of the channel state information (CSI).

\section{Conclusion}

This paper addresses the problem of estimating a channel supposed to follow a Rayleigh model with Jakes' Doppler spectrum (initially called the Clarke model) using a second order autoregressive model with Kalman filter (KF). Analytic results clearly show that the widely used $\operatorname{AR}(2)$ model tuned by the correlation matching $(\mathrm{CM})$ criterion is not accurate for low SNR and low to moderate Doppler frequencies $f_{d} T \leq 10^{-2}$. Therefore, we suggested switching to the minimum asymptotic variance (MAV) criterion to improve the estimation performance. We provided closed-form formulas for the optimal tuning of the $\operatorname{AR}(2)$ parameters and for the theoretical performance. Moreover, we also obtained some insight into the physical meaning and interpretations of the $\operatorname{AR}(2)$ parameters through the shape of the spectrum of the optimal AR(2) process. In particular, it has been shown that the dumping ratio of the spectrum of the $\mathrm{AR}(2)$ process should be tuned proportionally to the $\frac{1}{5}$ th power of the ratio of the Doppler frequency to the SNR. Simulation results show better MSE performance for the proposed AR(2)-MAV KF compared to $\mathrm{AR}(2)-\mathrm{KF}, \mathrm{AR}(1)-\mathrm{KF}$ and second-order LMS based algorithms of the literature, and similar performance compared to the much more complex Correlation Matching based $\operatorname{AR}(p)-\mathrm{KF}$ with order $p=15$. The performance is also equivalent to that of the second order Random Walk model-based Kalman filter of the literature, but with a stationary instead of unstationary 
state-space model to approximate the fading dynamic.

\section{Appendix A. Physical Parameters of AR(2)}

Appendix A.1. Proofs of (13) and (14)

The AR(2) model (4) is a stochastic difference equation. To establish the connection between the two pairs of parameters $\left\{f_{\mathrm{AR}(2)} T, r\right\}$ and the parameters of a second-order continuous-time (analog) deterministic model $\left\{f_{n, \mathrm{AR}(2)} T, \zeta_{\mathrm{AR}(2)}\right\}$, it is more convenient to consider the deterministic version of the $\operatorname{AR}(2)$ model given in the absence of state noise $\left(\sigma_{u}^{2}=0\right)$, which generates a damped cosine cycle. An expression for this cycle can be obtained by recursion, by using $a_{1}$ and $a_{2}$ as defined in (11). The $k$ th iteration of this recursion is

$$
\tilde{\alpha}_{(k)}=c \cdot r^{k} \cos \left(2 \pi f_{\mathrm{AR}(2)} k T\right),
$$

where $c$ is the amplitude of the cycle generated by the initial values of $\tilde{\alpha}_{(0)}$ and $\tilde{\alpha}_{(-1)}$. Indeed, A.1 can be proved recursively. Suppose it is true for a given iteration $k$. Then the $k+1$ th iteration is given by

$$
\begin{aligned}
\tilde{\alpha}_{(k+1)}=a_{1} \tilde{\alpha}_{(k)} & +a_{2} \tilde{\alpha}_{(k-1)} 2 c r^{k+1} \cos \left(2 \pi f_{\mathrm{AR}(2)} T\right) \cos \left(2 \pi f_{\mathrm{AR}(2)} k T\right) \\
& -c r^{k+1} \cos \left(2 \pi f_{\mathrm{AR}(2)}(k-1) T\right)=c r^{k+1} \cos \left(2 \pi f_{\mathrm{AR}(2)}(k+1) T\right) .
\end{aligned}
$$

This completes the recursive process since A.1 is also true for the initial iteration $k=1$.

On the other hand, the pair of parameters $\left\{f_{n, \mathrm{AR}(2)} T, \zeta_{\mathrm{AR}(2)}\right\}$ are the physical parameters of the second order filter in continuous time. Within the context 
of basic vibration theory [46] for continuous time, the general solution of the classical second order ordinary differential equation is of the form

$$
\tilde{\alpha}(t)=c \cdot e^{-\zeta_{\mathrm{AR}(2)} 2 \pi f_{n, \mathrm{AR}(2)} t} \cos \left(2 \pi f_{\mathrm{AR}(2)} t\right),
$$

where $t$ is continuous time. Sampling the above equation with a sampling period of $T$ yields

$$
\tilde{\alpha}_{(k)}=c \cdot e^{-\zeta_{\mathrm{AR}(2)} 2 \pi f_{n, \mathrm{AR}(2)} k T} \cos \left(2 \pi f_{\mathrm{AR}(2)} k T\right) .
$$

Comparing A.1 and A.3 yields $e^{-\zeta_{\mathrm{AR}(2)} 2 \pi f_{n, \mathrm{AR}(2)} T}=r$, which gives 14 .

Then, from [46], we have

$$
\left(2 \pi f_{\mathrm{AR}(2)} T\right)^{2}=\left(1-\zeta_{\mathrm{AR}(2)}^{2}\right)\left(2 \pi f_{n, \mathrm{AR}(2)} T\right)^{2} .
$$

Substituting for $\zeta_{\mathrm{AR}(2)}$ from (14) into A.4) yields (13).

\section{Appendix A.2. Proof of (44)}

Substituting $1-\delta$ for $r$ in (14) and using $\ln (1-\delta) \simeq-\delta$, which is justified by (iii), yields $\zeta_{\mathrm{AR}(2)} \simeq \frac{\delta}{\omega_{n, \mathrm{AR}(2)} T}$. Then, using 16 yields

$$
\zeta_{\mathrm{AR}(2)} \simeq \frac{\delta}{\omega_{\mathrm{AR}(2)} T}
$$

Applying (A.5) to $\delta=\delta_{(\mathrm{MAV})}$ gives 44 .

Appendix A.3. Proof of Assumption (vii)

As previously stated, $\zeta_{\mathrm{AR}(2)}$ should be $\ll 1$ in order to have a peak. In this case, from A.5,

$$
\begin{aligned}
& \delta \ll \omega_{\mathrm{AR}(2)} T \\
& \delta^{2} \ll \delta \omega_{\mathrm{AR}(2)} T
\end{aligned}
$$


and since $2 \sqrt{1-\delta}>1$ due to (iii)

$$
\delta^{2} \ll 2 \delta \sqrt{1-\delta} \omega_{\mathrm{AR}(2)} T
$$

Using Assumption (iii) yields $\delta^{2} \ll \delta \ll \sqrt{\delta}$, which results in

$$
\delta^{2} \ll 2 \sqrt{\delta(1-\delta)} \omega_{\mathrm{AR}(2)} T
$$

Then, combining Assumptions (ii) and (28) (which is obtained from Assumptions (i) and (iii) as explained in detail in Appendix H gives Assumption (vii).

\section{Appendix B. Exact Solutions for $P_{11}^{\prime}, K_{1}$ and $K_{2}$}

Appendix B.1. Solution for $P_{11}^{\prime}$ and $K_{1}$

Optimal linear control with quadratic performance criteria is widely used.

A non-recursive algebraic solution for the optimal gains is presented in [37].

This method can be used in our case for the determination of the steadystate Kalman filter. This solution allows determining the steady-state gains and covariance matrices directly, without iteration. In [37] it is shown that the solution of the filter equations $(20)$ and $(23)$ is obtained by finding the eigenvalues and eigenvectors of the following $4 \times 4$ matrix:

$$
\mathbf{H}_{\mathbf{f}}=\left[\begin{array}{cc}
\boldsymbol{\Phi}^{-T} & \boldsymbol{\Phi}^{-T} \mathbf{R}_{\mathbf{f}}^{\dagger} \\
\mathbf{Q}_{\mathbf{f}}^{*} \boldsymbol{\Phi}^{-T} & \boldsymbol{\Phi}+\mathbf{Q}_{\mathbf{f}}^{*} \boldsymbol{\Phi}^{-T} \mathbf{R}_{\mathbf{f}}^{\dagger}
\end{array}\right]
$$

where $\boldsymbol{\Phi}^{-T}=\mathbf{M}^{-T}, \mathbf{R}_{\mathbf{f}}^{\dagger}=\frac{\mathbf{s} \cdot \mathbf{s}^{T}}{\sigma_{w}^{2}}$, and $\mathbf{Q}_{\mathbf{f}}^{*}=\frac{\mathbf{s}^{T} \cdot \mathbf{s}}{\sigma_{u}^{2}}$

with $\mathbf{M}$ and $\mathbf{s}$ as defined in Section 3.1.

In [37] it is also shown that if $\lambda_{i}$ is an eigenvalue of $\mathbf{H}_{\mathbf{f}}$, then $1 / \lambda_{i}$ is also an 
eigenvalue, and the problem is reduced to finding two eigenvalues instead of four.

If $\lambda_{i}$ is an eigenvalue of $\mathbf{H}_{\mathbf{f}}$, the corresponding eigenvector can be found to be

$$
\mathbf{v}_{i}=\left[\begin{array}{c}
1 \\
a_{2} \lambda_{i} \\
\sigma_{w}^{2}\left(a_{2} \lambda_{i}\left(\lambda_{i}+a_{1} / a_{2}\right)-1\right) \\
\left(\sigma_{w}^{2}\left(a_{2} \lambda_{i}\left(\lambda_{i}+a_{1} / a_{2}\right)-1\right)\right) / \lambda_{i}
\end{array}\right] .
$$

The steady state solution for $\mathbf{P}_{\infty}^{\prime}$ is then given by [37]

$$
\mathbf{P}_{\infty}^{\prime}=\mathbf{W}_{21} \mathbf{W}_{11}^{-1},
$$

where $\mathbf{W}_{21}$ and $\mathbf{W}_{11}$ are defined as follows:

Put $\mathbf{W}$ to be the $4 \times 4$ matrix formed from the eigenvectors $\mathbf{v}_{1}, \mathbf{v}_{2}, \mathbf{v}_{1}^{\prime}$ and $\mathbf{v}_{2}{ }^{\prime}$ and their corresponding eigenvalues $\lambda_{1}, \lambda_{2}, 1 / \lambda_{1}$ and $1 / \lambda_{2}$ : $\mathbf{W}=$ $\left[\mathbf{v}_{1}, \mathbf{v}_{2}, \mathbf{v}_{1}^{\prime}, \mathbf{v}_{2}^{\prime}\right]=\left[\begin{array}{ll}\mathbf{W}_{11} & \mathbf{W}_{12} \\ \mathbf{W}_{21} & \mathbf{W}_{22}\end{array}\right]$ with $\mathbf{W}_{11}, \mathbf{W}_{12}, \mathbf{W}_{21}$ and $\mathbf{W}_{22}$ being the $2 \times 2$ matrices

$$
\begin{aligned}
& \mathbf{W}_{11}=\left[\begin{array}{cc}
1 & 1 \\
a_{2} \lambda_{1} & a_{2} \lambda_{2}
\end{array}\right] \\
& \mathbf{W}_{21}=\left[\begin{array}{cc}
x & y \\
x / \lambda_{1} & y / \lambda_{2}
\end{array}\right],
\end{aligned}
$$

where $x=\sigma_{w}^{2}\left(a_{2} \lambda_{1}\left(\lambda_{1}+\frac{a_{1}}{a_{2}}\right)-1\right)$ and $y=\sigma_{w}^{2}\left(a_{2} \lambda_{2}\left(\lambda_{2}+\frac{a_{1}}{a_{2}}\right)-1\right)$. 
Using this method, we have a matrix $\mathbf{P}_{\infty}^{\prime}$ from which we can extract $P_{11}^{\prime}$ :

$$
P_{11}^{\prime}=-\sigma_{w}^{2}\left(1+a_{2} \lambda_{1} \lambda_{2}\right)
$$

Therefore it remains to calculate the product $\lambda_{1} \lambda_{2}$ to find $P_{11}^{\prime}$. The method proposed in [47] is based on the characteristic polynomial of the matrix $\mathbf{H}_{\mathbf{f}}$. An expression for the characteristic polynomial $\mathrm{Pol}_{1}$ is evaluated by calculating the determinant of the matrix $\left|\mathbf{H}_{\mathbf{f}}-\lambda \mathbf{I}\right|$, where $\mathbf{I}$ is the $4 \times 4$ identity matrix. The corresponding characteristic polynomial is

$$
\mathrm{Pol}_{1}=\lambda^{4}-a \lambda^{3}+(b+2) \lambda^{2}-a \lambda+1
$$

where

$$
\begin{aligned}
a & =\frac{a_{1} a_{2}-a_{1}}{a_{2}} \\
b & =-\frac{a_{1}^{2}+a_{2}^{2}+\sigma_{u}^{2} / \sigma_{w}^{2}+1}{a_{2}}-2 .
\end{aligned}
$$

In addition, because of the fact that the inverse of an eigenvalue is also an eigenvalue, there must be another expression for the characteristic polynomial of the following form:

$$
\begin{aligned}
\mathrm{Pol}_{2}= & \left(\lambda-\lambda_{1}\right)\left(\lambda-\lambda_{2}\right)\left(\lambda-1 / \lambda_{1}\right)\left(\lambda-1 / \lambda_{2}\right) \\
= & \lambda^{4}-\left(\lambda_{1}+\lambda_{2}+1 / \lambda_{1}+1 / \lambda_{2}\right) \lambda^{3}+ \\
& \left(\left(\lambda_{1}+1 / \lambda_{1}\right)\left(\lambda_{2}+1 / \lambda_{2}\right)+2\right) \lambda^{2} \\
& -\left(\lambda_{1}+\lambda_{2}+1 / \lambda_{1}+1 / \lambda_{2}\right) \lambda+1 .
\end{aligned}
$$

Identifying (B.10) and (B.7) we get

$$
\begin{aligned}
& a=\lambda_{1}+\lambda_{2}+1 / \lambda_{1}+1 / \lambda_{2} . \\
& b=\left(\lambda_{1}+1 / \lambda_{1}\right)\left(\lambda_{2}+1 / \lambda_{2}\right) .
\end{aligned}
$$


Equation B.11 can be rewritten as

$$
\lambda_{1}+\lambda_{2}=a \frac{\lambda_{1} \lambda_{2}}{\lambda_{1} \lambda_{2}+1} .
$$

Thus we have a relation between the sum and the product of the eigenvalues. Now developing the product in (B.12) and using (B.13) yields

$$
b=\lambda_{1} \lambda_{2}+\frac{1}{\lambda_{1} \lambda_{2}}+\frac{a^{2}}{\lambda_{1} \lambda_{2}+\frac{1}{\lambda_{1} \lambda_{2}}+2}-2 .
$$

Put $d=\lambda_{1} \lambda_{2}+\frac{1}{\lambda_{1} \lambda_{2}}$. Equation B.14 is a second-order equation in $d$ with solution

$$
d=\frac{1}{2}\left(b+\sqrt{(b+4)^{2}-4 a^{2}}\right) .
$$

Moreover, $d=\lambda_{1} \lambda_{2}+\frac{1}{\lambda_{1} \lambda_{2}}$ is a second-order equation in $\lambda_{1} \lambda_{2}$, hence with the solution

$$
\lambda_{1} \lambda_{2}=\frac{1}{2}\left(d+\sqrt{d^{2}-4}\right),
$$

with $d$ given in (B.15), $b$ in (B.9), and $a$ in (B.8). To sum up, an exact expression for $K_{1}$ is given by (24) where $P_{11}^{\prime}$ was given in B.6. and $\lambda_{1} \lambda_{2}$ in (B.16).

It remains now to calculate $K_{2}$. In the following, an expression for $K_{2}$ is obtained after some direct manipulations of the Riccati equations.

Appendix B.2. Expression for $K_{2}$

In this appendix an expression for $K_{2}$ is calculated. It is known that $\operatorname{cov}(x, y)=$ $\operatorname{cov}(y, x)$, where $\operatorname{cov}(x, y)$ is the covariance of the two variables $x$ and $y$. That is why $P_{12}=P_{21}$, where $P_{12}$ and $P_{21}$ are the elements of the posteriori error covariance matrix defined in 27 ). The same is true for $P_{12}^{\prime}=P_{21}^{\prime}$, where $P_{12}^{\prime}$ 
and $P_{21}^{\prime}$ are the elements of the predicted error covariance matrix defined in (26).

Then from (27) and by comparing the expressions for $P_{12}$ and $P_{21}$ we get $\left(1-K_{1}\right) P_{12}^{\prime}+K_{2} P_{11}^{\prime}=P_{12}^{\prime}$, yielding

$$
P_{12}^{\prime}=\frac{K_{2} P_{11}^{\prime}}{K_{1}} .
$$

Also from 27), $P_{12}=\left(1-K_{1}\right) P_{12}^{\prime}$ and $P_{11}=\left(1-K_{1}\right) P_{11}^{\prime}$. By substituting these equations into (B.17), we get

$$
P_{12}=\frac{K_{2} P_{11}}{K_{1}} .
$$

Another expression for $P_{12}$ could be deduced from (26) and (27), using $P_{12}^{\prime}=$ $a_{1} P_{11}+a_{2} P_{21}$ and $P_{12}=\left(1-K_{1}\right) P_{12}^{\prime}$ is

$$
P_{12}=\frac{a_{1}\left(1-K_{1}\right) P_{11}}{1-a_{2}+a_{2} K_{1}} .
$$

By comparing Eqs $(\overline{B .18})$ and $(\overline{B .19})$, an exact expression for $K_{2}$ is obtained in terms of $K_{1}, a_{1}$ and $a_{2}$ :

$$
K_{2}=\frac{a_{1}\left(1-K_{1}\right) K_{1}}{1-a_{2}+a_{2} K_{1}} .
$$

\section{Appendix C. Approximation for $K_{1}$ : Proof of 29}

The aim of this section is to find approximate expressions for Eqs (B.8), (B.9), (B.15), and (B.16), given in Section Appendix B.1, in order to obtain the approximate expression $(29)$ for $K_{1}$. The approach we follow is to work with the pair of parameters $\left\{f_{\mathrm{AR}(2)} T, r=1-\delta\right\}$ instead of $\left\{a_{1}, a_{2}\right\}$ to exploit the assumptions of Section 3.3.1. 
Appendix C.1. Approximation for a B.8

Using (11) and $r=1-\delta$ gives

$$
a=\frac{2 \cos \left(\omega_{\mathrm{AR}(2)} T\right)\left(1+(1-\delta)^{2}\right)}{(1-\delta)} .
$$

It can be deduced from (i) and (iii) that $\frac{1}{1-\delta} \simeq 1+\delta+\delta^{2}$ and $\delta^{3} \ll \delta^{2} \ll$ $\delta \ll 1$, so

$$
\begin{aligned}
a & \simeq 2 \cos \left(\omega_{\mathrm{AR}(2)} T\right)\left(2-2 \delta+\delta^{2}\right)\left(1+\delta+\delta^{2}\right) \\
& \simeq 2 \cos \left(\omega_{\mathrm{AR}(2)} T\right)\left(2+\delta^{2}-\delta^{3}+\delta^{4}\right) \\
& \simeq 2 \cos \left(\omega_{\mathrm{AR}(2)} T\right)\left(2+\delta^{2}\right) .
\end{aligned}
$$

Appendix C.2. Approximation for $b$ B.9

$$
\begin{aligned}
b & =\frac{-a_{1}^{2}}{a_{2}}-a_{2}-\frac{1}{a_{2}}-\frac{\sigma_{u}^{2}}{\sigma_{w}^{2} a_{2}}-2 \\
& =\frac{-4 r^{2} \cos ^{2}\left(\omega_{\mathrm{AR}(2)} T\right)}{-r^{2}}+r^{2}+\frac{1}{r^{2}}+\frac{\sigma_{u}^{2}}{\sigma_{w}^{2} r^{2}}-2 \\
& \simeq 4 \cos \left(\omega_{\mathrm{AR}(2)} T\right)^{2}+(1-\delta)^{2}+\frac{\sigma_{u}^{2}+\sigma_{w}^{2}}{\sigma_{w}^{2}(1-\delta)^{2}}-2 .
\end{aligned}
$$

From (iii) we can use $\frac{1}{(1-\delta)^{2}}=\sum_{n=0}^{\infty}(n+1) \delta^{n} \simeq 1+2 \delta+3 \delta^{2}$, yielding

$$
b \simeq 4 \cos \left(\omega_{\mathrm{AR}(2)} T\right)^{2}+\frac{\sigma_{u}^{2}\left(1+2 \delta+3 \delta^{2}\right)}{\sigma_{w}^{2}}+4 \delta^{2} .
$$

Again, we use (iii), resulting in $\frac{3 \sigma_{u}^{2} \delta^{2}}{\sigma_{w}^{2}} \ll \frac{2 \sigma_{u}^{2} \delta}{\sigma_{w}^{2}} \ll \frac{\sigma_{u}^{2}}{\sigma_{w}^{2}}$, and next (v) to deduce

$$
b \simeq 4 \cos \left(\omega_{\mathrm{AR}(2)} T\right)^{2}+\frac{\sigma_{u}^{2}}{\sigma_{w}^{2}}+4 \delta^{2} .
$$


Appendix C.3. Approximation for d B.15)

We use the notation $\mu=(b+4)^{2}-4 a^{2}$. From $(\mathrm{i})$, we have $\cos \left(\omega_{\mathrm{AR}(2)} T\right) \simeq 1-$ $\frac{\left(\omega_{\mathrm{AR}(2)} T\right)^{2}}{2}$. Using $\mathrm{C} .1$ and $\mathrm{C} .4$ together with the previous approximation yields

$$
\mu \simeq\left(4 \cos \left(\omega_{\mathrm{AR}(2)} T\right)^{2}+\frac{\sigma_{u}^{2}}{\sigma_{w}^{2}}+4 \delta^{2}+4\right)^{2}-16 \cos \left(\omega_{\mathrm{AR}(2)} T\right)^{2}\left(2+\delta^{2}\right)^{2} .
$$

However, by using the double-angle trigonometric identity and Assumption (i) we have $\cos \left(\omega_{\mathrm{AR}(2)} T\right)^{2}=\frac{1+\cos \left(2 \omega_{\mathrm{AR}(2)} T\right)}{2} \simeq \frac{1}{2}\left(1+\left(1-\frac{\left(2 \omega_{\mathrm{AR}(2)} T\right)^{2}}{2}\right)\right) \simeq$ $1-\left(\omega_{\mathrm{AR}(2)} T\right)^{2}$, and so

$$
\begin{aligned}
\mu & \simeq 16\left(\omega_{\mathrm{AR}(2)} T\right)^{4}+\left(16 \delta^{4}+32 \delta^{2}-8 \frac{\sigma_{u}^{2}}{\sigma_{w}^{2}}\right)\left(\omega_{\mathrm{AR}(2)} T\right)^{2} \\
& +\frac{\sigma_{u}^{4}}{\sigma_{w}^{4}}+16 \frac{\sigma_{u}^{2}}{\sigma_{w}^{2}}+8 \delta^{2} \frac{\sigma_{u}^{2}}{\sigma_{w}^{2}} .
\end{aligned}
$$

Assumptions (i), (iii) and (v) lead to $\left(\omega_{\mathrm{AR}(2)} T\right)^{4} \ll\left(\omega_{\mathrm{AR}(2)} T\right)^{2} \ll 1, \delta^{4} \ll$ $\delta^{2} \ll \delta \ll 1$ and $\frac{\sigma_{u}^{4}}{\sigma_{w}^{4}} \ll \frac{\sigma_{u}^{2}}{\sigma_{w}^{2}} \ll 1$, respectively. Thus, $\mu$ can be approximated by $\mu \simeq 16 \frac{\sigma_{u}^{2}}{\sigma_{w}^{2}}$. From this equation we can write

$$
\begin{aligned}
d & \simeq \frac{1}{2}\left(4 \cos \left(\omega_{\mathrm{AR}(2)} T\right)^{2}+\frac{\sigma_{u}^{2}}{\sigma_{w}^{2}}+4 \delta^{2}+\sqrt{\mu}\right) \\
& \simeq \frac{1}{2}\left(4\left(1-\left(\omega_{\mathrm{AR}(2)} T\right)^{2}\right)+\frac{\sigma_{u}^{2}}{\sigma_{w}^{2}}+4 \delta^{2}+4 \frac{\sigma_{u}}{\sigma_{w}}\right) .
\end{aligned}
$$

Using (v), (vi), and (vii) results in

$$
d \simeq 2+2 \frac{\sigma_{u}}{\sigma_{w}} .
$$

Appendix C.4. Approximation for $\lambda_{1} \lambda_{2}$ B.16

Substituting (C.7) into B.16) gives

$$
\lambda_{1} \lambda_{2} \simeq 1+\frac{\sigma_{u}}{\sigma_{w}}+\frac{1}{2} \sqrt{4+8 \frac{\sigma_{u}}{\sigma_{w}}+4 \frac{\sigma_{u}^{2}}{\sigma_{w}^{2}}-4} .
$$


Assumption $(\mathrm{v})$ results in $\frac{\sigma_{u}^{2}}{\sigma_{w}^{2}} \ll \frac{\sigma_{u}}{\sigma_{w}} \ll \sqrt{\frac{\sigma_{u}}{\sigma_{w}}}$ :

$$
\begin{aligned}
& \lambda_{1} \lambda_{2} \simeq 1+\frac{\sigma_{u}}{\sigma_{w}}+\sqrt{\frac{2 \sigma_{u}}{\sigma_{w}}} \\
& \lambda_{1} \lambda_{2} \simeq 1+\sqrt{\frac{2 \sigma_{u}}{\sigma_{w}}}
\end{aligned}
$$

Appendix C.5. Approximation for $P_{11}^{\prime}$ B.6

Substituting (C.8) into B.6) gives

$$
P_{11}^{\prime} \simeq-\sigma_{w}^{2}\left(1-(1-\delta)^{2}\left(1+\sqrt{\frac{2 \sigma_{u}}{\sigma_{w}}}\right)\right)
$$

Using $\delta^{2} \ll \delta \ll 1$ from (iii) together with (vii) yields

$$
P_{11}^{\prime} \simeq \sigma_{w}^{2} \sqrt{\frac{2 \sigma_{u}}{\sigma_{w}}}
$$

Appendix C.6. Approximation for $K_{1}$ (24)

First, we have $P_{11}^{\prime} \ll \sigma_{w}^{2}$, which, in turn, using $\sqrt{24}$ yields $K_{1} \simeq \frac{\mathrm{P}_{11}^{\prime}}{\sigma_{w}^{2}}$. From the previous equation and (C.9), we obtain 29.

\section{Appendix D. Approximation for $K_{2}(\mathrm{~B} .20)$ :}

The aim of this the section is to find an approximate expression of (B.20) already established in Appendix B.2 in terms of $K_{1}$. The approximation used in this section is based on the pair of parameters $\left\{f_{\mathrm{AR}(2)} T, r=1-\delta\right\}$ instead of $\left\{a_{1}, a_{2}\right\}$. Using 11 and $r=1-\delta$ in B.20 gives

$$
K_{2}=\frac{2(1-\delta) \cos \left(\omega_{\mathrm{AR}(2)} T\right)\left(1-K_{1}\right) K_{1}}{1+(1-\delta)^{2}-(1-\delta)^{2} K_{1}}
$$


From (i), we have $\cos \left(\omega_{\mathrm{AR}(2)} T\right) \simeq 1-\frac{\left(\omega_{\mathrm{AR}(2)} T\right)^{2}}{2}$. The expression in D.1) becomes

$$
K_{2} \simeq \frac{2(1-\delta)\left(1-\frac{\left(\omega_{\mathrm{AR}(2)} T\right)^{2}}{2}\right)\left(1-K_{1}\right) K_{1}}{1+(1-\delta)^{2}-(1-\delta)^{2} K_{1}}
$$

Assumptions (i), (iii) and (viii) lead to $\left(\omega_{\mathrm{AR}(2)} T\right)^{2} \ll\left(\omega_{\mathrm{AR}(2)} T\right) \ll 1, \delta^{2} \ll$ $\delta \ll 1$ and $K_{1}^{2} \ll K_{1} \ll 1$, respectively. Thus, (D.2) can be approximated by

$$
K_{2} \simeq K_{1}
$$

\section{Appendix E. Expression for $L(z)$ : Proof of $(31)$}

Equations $(19)$ and $(22)$ of the Kalman filter, taken in the steady-state mode, give

$$
\begin{aligned}
\hat{\alpha}_{(k \mid k-1)} & =a_{1} \hat{\alpha}_{(k-1 \mid k-1)}+a_{2} \hat{\alpha}_{(k-2 \mid k-1)} \\
\hat{\alpha}_{(k \mid k)} & =\hat{\alpha}_{(k \mid k-1)}+K_{1}\left(y_{(k)}-\hat{\alpha}_{(k \mid k-1)}\right) \\
\hat{\alpha}_{(k-1 \mid k)} & =\hat{\alpha}_{(k-1 \mid k-1)}+K_{2}\left(y_{(k)}-\hat{\alpha}_{(k \mid k-1)}\right) .
\end{aligned}
$$

We define the error signal as the difference between the observation and the prediction:

$$
v_{\epsilon(k)}=y_{(k)}-\hat{\alpha}_{(k \mid k-1)} .
$$

By substituting $($ E.1) and $($ E.3 into E.2 we have

$$
\hat{\alpha}_{(k \mid k)}=a_{1} \hat{\alpha}_{(k-1 \mid k-1)}+a_{2}\left(\hat{\alpha}_{(k-2 \mid k-2)}+K_{2} v_{\epsilon(k-1)}\right)+K_{1} v_{\epsilon(k)} .
$$

Then, applying the $z$-transformation gives

$$
\hat{\alpha}(z)\left[1-a_{1} z^{-1}-a_{2} z^{-2}\right]=\left[a_{2} K_{2} z^{-1}+K_{1}\right] v_{\epsilon}(z) .
$$


Using $\hat{\alpha}_{(k \mid k-1)}=\hat{\alpha}_{(k \mid k)}-K_{1} v_{\epsilon(k)}$ from E.2 in (E.4) gives

$$
v_{\epsilon(k)}=y_{(k)}-\hat{\alpha}_{(k \mid k)}+K_{1} v_{\epsilon(k)} .
$$

Taking the $z$-transform yields

$$
v_{\epsilon}(z)=\frac{y(z)-\hat{\alpha}(z)}{1-K_{1}}
$$

Substituting (E.7) into (E.5), we can find an expression for the closed-loop transfer function $L(z)=\frac{\hat{\alpha}(z)}{y(z)}$, which leads to 31 .

\section{Appendix F. Expressions and approximations for $\omega_{n, L(z)} T$ and $\zeta_{L(z)}$}

The aim of this appendix is to establish the expressions and the approximations for the normalized natural frequency $\omega_{n, L(z)} T$ and the damping ratio $\zeta_{L(z)}$ of the transfer function of $\mathrm{KF}, L(z)$ given in 31 .

Appendix F.1. Expressions for $\omega_{n, L(z)} T$ and $\zeta_{L(z)}$

The transfer function of a continuous-time second order systems as a function of the natural frequency and the damping ratio is [34]:

$$
G(s)=\frac{\omega_{n}^{2}}{s^{2}+2 \zeta \omega_{n} s+\omega_{n}^{2}}
$$

Using $z=\mathrm{e}^{j 2 \pi f T}$ and then $z^{-1} \simeq 1-j 2 \pi f T$ for $f T \ll 1$ in (31), and comparing the obtained expression with the transfer function in (F.1), we obtain

$$
\begin{aligned}
\left(\omega_{n, L(z)} T\right)^{2} & =\frac{K_{1}\left(a_{1}+a_{2}\right)+K_{2} a_{2}+1-a_{1}-a_{2}}{-a_{2}\left(1-K_{1}\right)} \\
\left(2 \zeta_{L(z)} \omega_{n, L(z)} T\right) & =\frac{K_{1}\left(-a_{1}-2 a_{2}\right)-a_{2} K_{2}+a_{1}+2 a_{2}}{-a_{2}\left(1-K_{1}\right)}
\end{aligned}
$$


From (F.2) and (F.3), the expressions for $\left(\omega_{n, L(z)} T\right)$ and $\zeta_{L(z)}$ are deduced in terms of $a_{1}, a_{2}, K_{1}$ and $K_{2}$. However the goal is to obtain approximate expressions for $\left(\omega_{n, L(z)} T\right)$ and $\zeta_{L(z)}$.

Appendix F.2. Approximate expressions for $\omega_{n, L(z)} T$ and $\zeta_{L(z)}$

In this section the approximate expressions for $\left(\omega_{n, L(z)} T\right)$ and $\zeta_{L(z)}$ are provided. The approximations used in this section are based on the pair of parameters $\left\{f_{\mathrm{AR}(2)} T, r=1-\delta\right\}$ instead of $\left\{a_{1}, a_{2}\right\}$. Using (11) and $r=1-\delta$ in $(\mathrm{F} .2)$ and $(\mathrm{F} .3)$ gives

$$
\begin{gathered}
K_{1}\left(2(1-\delta) \cos \left(\omega_{\mathrm{AR}(2)} T\right)-\left(1-\delta^{2}\right)\right) \\
\left(\omega_{L(z)} T\right)^{2} \simeq \frac{-K_{2}(1-\delta)^{2}+1-2(1-\delta) \cos \left(\omega_{\mathrm{AR}(2)} T\right)+(1-\delta)^{2}}{(1-\delta)^{2}\left(1-K_{1}\right)} \\
\left(2 \zeta_{L(z)} \omega_{L(z)} T\right) \simeq \frac{(1-\delta)^{2} K_{2}+2(1-\delta) \cos \left(\omega_{\mathrm{AR}(2)} T\right)}{\times\left(1-K_{1}\right)-2(1-\delta)^{2}\left(1-K_{1}\right)}
\end{gathered}
$$

Appendix F.2.1. Approximate expression for $\omega_{L(z)} T$

Replacing $K_{2}$ (Eq. (B.20) ) by its expression in (F.4) and referring to Assumptions (i), (iii) and (viii) that lead respectively to $\cos \left(\omega_{\mathrm{AR}(2)} T\right) \simeq 1-$ $\frac{\left(\omega_{\mathrm{AR}(2)} T\right)^{2}}{2},\left(f_{\mathrm{AR}(2)} T\right)^{2} \ll f_{\mathrm{AR}(2)} T \ll 1, \delta^{2} \ll \delta \ll 1$ and $K_{1}^{2} \ll K_{1} \ll 1$, we have

$$
\left(\omega_{n, L(z)} T\right)^{2} \simeq K_{1}-\frac{K_{1}\left(1-\delta-K_{1}\right)}{1-\delta-\frac{K_{1}}{2}} .
$$

and since $K_{1} \ll 1$ and $\delta \ll K_{1}$, from (viii) and (ix) respectively, we have

$$
\left(\omega_{n, L(z)} T\right)^{2} \simeq \frac{K_{1}^{2}}{2} .
$$

In this case

$$
\omega_{n, L(z)} T \bumpeq \frac{K_{1}}{\sqrt{2}} .
$$


Appendix F.2.2. Approximate expression for $\zeta_{L(z)}$

In this section we provide an approximate expression for $\zeta_{L(z)}$. In addition to all the assumptions used in the previous section, we use the approximation $K_{2} \simeq K_{1}($ see $(\mathrm{D} .1))$, and then (F.5) becomes

$$
\begin{aligned}
\left(2 \zeta_{L(z)} \omega_{n, L(z)} T\right) & \simeq \frac{-2 K_{1}\left(\delta-\left(\omega_{\mathrm{AR}(2)} T\right)^{2}\right)+(1-2 \delta) K_{2}}{+2\left(\delta-\left(\omega_{\mathrm{AR}(2)} T\right)^{2}\right)} \\
& \simeq \frac{K_{2}}{1-2 \delta-K_{1}} \\
& \simeq K_{1} .
\end{aligned}
$$

From (F.8) and (F.11),

$$
\zeta_{L(z)} \simeq \frac{\sqrt{2}}{2}
$$

\section{Appendix G. Calculation and approximation for $\sigma_{\epsilon w}^{2}$ : Proof of $(34)$}

In this appendix, the goal is to calculate and approximate the static error

variance $\sigma_{\epsilon w}^{2}$ due to the observation noise variance $\sigma_{w}^{2}$, which can be rewritten

$$
\sigma_{\epsilon w}^{2} \stackrel{\text { def }}{=} \sigma_{w}^{2} T \int_{-\frac{1}{2 T}}^{\frac{1}{2 T}}\left|L\left(e^{2 i \pi f T}\right)\right|^{2} d f=\sigma_{w}^{2} \times B N W
$$

where $B N W$ is the normalized equivalent noise bandwidth of the system, defined by

$$
B N W=T \int_{-\frac{1}{2 T}}^{\frac{1}{2 T}}\left|L\left(e^{2 i \pi f T}\right)\right|^{2} d f .
$$

An exact expression for $B N W$ can be derived by using the method presented in [48]. It can be evaluated analytically by solving a system of equations in 
matrix form. The elements of this matrix are the coefficients of the numerator and denominator of the integrand $L(z)$. Now $L(z)$ can be written

$$
L(z)=\frac{B_{0} z^{2}+B_{1} z+B_{2}}{A_{0} z^{2}+A_{1} z+A_{2}},
$$

where $A_{0}=1, A_{1}=a_{2} K_{2}-a_{1}\left(1-K_{1}\right), A_{2}=-a_{2}\left(1-K_{1}\right), B_{0}=K_{1}$, $B_{1}=K_{2} a_{2}$, and $B_{2}=0$,

and the corresponding matrix equation is

$$
\left[\begin{array}{ccc}
A_{0} & A_{1} & A_{2} \\
A_{1} & A_{0}+A_{2} & A_{1} \\
A_{2} & 0 & A_{2}
\end{array}\right] \times\left[\begin{array}{c}
A_{0} B N W \\
M_{1} \\
M_{2}
\end{array}\right]=\left[\begin{array}{c}
B_{0}^{2}+B_{1}^{2}+B_{2}^{2} \\
2\left(B_{0} B_{1}+B_{1} B_{2}\right) \\
2 B_{0} B_{2}
\end{array}\right],
$$

where $M_{1}$ and $M_{2}$ are evaluated in terms of $B N W, A_{0}, A_{1}, A_{2}, B_{0}, B_{1}$ and $B_{2}$. Replacing $K_{2}$ by its expression defined in $(\mathrm{B} .20)$, the calculation result of (G.4) leads to the following exact calculation in terms of $K_{1}, a_{1}$ and $a_{2}$,

$$
B N W=\frac{A K_{1}^{5}+B K_{1}^{4}+C K_{1}^{3}+D K_{1}^{2}}{E K_{1}^{5}+F K_{1}^{4}+G K_{1}^{3}+H K_{1}^{2}+I K_{1}+J},
$$


where

$$
\begin{aligned}
& A=a_{1}^{2} a_{2}^{3}+a_{2}^{3} \\
& B=-3 a_{1}^{2} a_{2}^{3}-a_{1}^{2} a_{2}^{2}+2 a_{1}^{2} a_{2}-3 a_{2}^{3}+3 a_{2}^{2} \\
& C=3 a_{1}^{2} a_{2}^{3}+2 a_{1}^{2} a_{2}^{2}-4 a_{1}^{2} a_{2}+3 a_{2}^{3}-6 a_{2}^{2}+3 a_{2} \\
& D=-a_{1}^{2} a_{2}^{3}-a_{1}^{2} a_{2}^{2}+2 a_{1}^{2} a_{2}-a_{2}^{3}+3 a_{2}^{2}-3 a_{2}+1 \\
& E=-a_{2}^{5} \\
& F=5 a_{2}^{5}-3 a_{2}^{4} \\
& G=a_{1}^{2} a_{2}^{3}-2 a_{1}^{2} a_{2}^{2}+a_{1}^{2} a_{2}-10 a_{2}^{5}+12 a_{2}^{4}-2 a_{2}^{3} \\
& H=-3 a_{1}^{2} a_{2}^{3}+5 a_{1}^{2} a_{2}^{2}-a_{1}^{2} a_{2}-a_{1}^{2}+10 a_{2}^{5}-18 a_{2}^{4}+6 a_{2}^{3}+2 a_{2}^{2} \\
& I=3 a_{1}^{2} a_{2}^{3}-4 a_{1}^{2} a_{2}^{2}-a_{1}^{2} a_{2}+2 a_{1}^{2}-5 a_{2}^{5}+12 a_{2}^{4}-6 a_{2}^{3}-4 a_{2}^{2}+3 a_{2} \\
& J=-a_{1}^{2} a_{2}^{3}+a_{1}^{2} a_{2}^{2}+a_{1}^{2} a_{2}-a_{1}^{2}+a_{2}^{5}-3 a_{2}^{4}+2 a_{2}^{2}+2 a_{2}^{3}-3 a_{2}+1 .
\end{aligned}
$$

The above exact expressions are next approximated using Assumptions (i), (ii), (iii), (iv), (v), (viii) defined in Section 3.3.1 and the expressions for $a_{1}$ and $a_{2}$ formulated in (11). In addition, we use the approximation $\cos \left(2 \pi f_{\mathrm{AR}(2)} T\right) \simeq$ 


$$
\begin{aligned}
1-\frac{\left(2 \pi f_{\mathrm{AR}(2)} T\right)^{2}}{2} \text { and }(1-\delta)^{n} & \simeq 1-n \delta, \text { so } \\
A & \simeq 32 \delta-12\left(2 \pi f_{\mathrm{AR}(2)} T\right)^{2} \\
B & \simeq 6-22 \delta \\
C & \simeq 32 \delta-12\left(2 \pi f_{\mathrm{AR}(2)} T\right)^{2} \\
D & \simeq 8\left(2 \pi f_{\mathrm{AR}(2)} T\right)^{2} \\
E & \simeq-1+10 \delta \\
F & \simeq-8+74 \delta \\
G & \simeq-112 \delta+8 \\
H & \simeq 48 \delta \\
I & \simeq 64 \delta^{2} \\
J & \simeq 32 \delta\left(2 \pi f_{\mathrm{AR}(2)} T\right)^{2} .
\end{aligned}
$$

The above approximations allow expressing $B N W$ in terms of the pair of $\mathrm{AR}(2)$ parameters $f_{\mathrm{AR}(2)} T, r=1-\delta$. By replacing each term by its approximation in G.5 and using the previous assumptions,

$$
\begin{aligned}
B N W & \simeq \frac{6 K_{1}^{4}+32 \delta K_{1}}{8 K_{1}^{3}+48 \delta K_{1}^{2}} \\
& \simeq \frac{6 K_{1}^{3}\left(K_{1}+\frac{32}{6} \delta\right)}{8 K_{1}^{2}\left(K_{1}+6 \delta\right)} \\
& \simeq \frac{3}{4} K_{1}
\end{aligned}
$$

using that $\frac{K_{1}+\frac{32}{6} \delta}{K_{1}+6 \delta} \simeq 1$, which can be assumed from $(\mathrm{v})$ 
Appendix G.1. Calculation and expression for (35)

The aim of this appendix is to calculate and approximate the dynamic error variance $\sigma_{\epsilon \alpha}^{2}$. This variance is due to the variations of $\alpha_{(k)}$ filtered by the high pass filter $1-L(z)$ :

$$
\sigma_{\epsilon \alpha}^{2} \stackrel{\text { def }}{=} \int_{-f_{d}}^{+f_{d}}\left|1-L\left(e^{2 i \pi f T}\right)\right|^{2} \Gamma_{\alpha}(f) d f .
$$

In order to obtain the result of the integration in (G.17), the term $\mid 1-$ $\left.L\left(e^{2 i \pi f T}\right)\right|^{2}$ needs to be calculated in the frequency range $\left[-f_{d}, f_{d}\right]$. To do so, we first substitute (31) for $L$ and we develop the calculation assuming low normalized frequencies, which leads to $z=e^{2 i \pi f T} \simeq 1+i 2 \pi f T$. The details of the calculation are provided in the on-line report [49]. The obtained expression is not tractable and needs further approximations. As the dominating values of the Jakes' spectrum are located in the vicinity of $\left\{-f_{d}, f_{d}\right\}$, we employ an approximation for $|1-L|^{2}$ valid in this range of frequencies. We use Assumptions (i) (viii) of Section 3.3.1 and the fact that it can be deduced from (viii) that $K_{1}^{4} \ll K_{1}^{3} \ll K_{1}^{2} \ll K_{1} \ll 1$, and from (i) and (iii) that $f_{d} T^{4} \ll f_{d} T^{3} \ll f_{d} T^{2} \ll f_{d} T \ll 1$. After some manipulations, we find $\left|1-L\left(e^{2 i \pi f T}\right)\right|^{2} \simeq \frac{(2 \pi f T)^{4}}{K_{1}^{4}}$ for $f$ in the vicinity of $f_{d},-f_{d}$ (the details of the calculation can be found in [49]). The integration is then computed from $\Gamma_{\alpha}(f)$ defined in (2) by changing variables, replacing $\left(f / f_{d}\right)$ by $\cos (\theta)$ :

$$
\sigma_{\epsilon \alpha}^{2} \simeq \int_{-f_{d}}^{+f_{d}} \frac{(2 \pi f T)^{4}}{K_{1}^{4}} \Gamma_{\alpha}(f) d f=\sigma_{\alpha}^{2} \frac{6 \pi^{4}\left(f_{d} T\right)^{4}}{K_{1}^{4}},
$$

which is equivalent to (35) 


\section{Appendix H. Approximation for $\sigma_{u}^{2}$}

In this section, an approximate expression for $\sigma_{u}^{2}$ is given. Replacing $r$ by $1-\delta$ in the expression (11) for $a_{2}$ yields

$$
a_{2}=-(1-\delta)^{2} \simeq-\left(1-2 \delta+\delta^{2}\right) \simeq-(1-2 \delta) .
$$

Supposing that $\delta^{2} \ll \delta$ (from Assumption [iii), we have

$$
\begin{aligned}
& 1+a_{2} \simeq 2 \delta \\
& 1-a_{2} \simeq(1-\delta) .
\end{aligned}
$$

By inserting the above equations into the expression for $\sigma_{u}^{2}$ defined in (8),

$$
\begin{aligned}
\sigma_{u}^{2} & \simeq \sigma_{\alpha}^{2} \frac{2 \delta}{2(1-\delta)}\left(2(1-\delta)-2(1-\delta) \cos \left(2 \pi f_{\mathrm{AR}(2)} T\right)\right) \\
& \times\left(2(1-\delta)+2(1-\delta) \cos \left(2 \pi f_{\mathrm{AR}(2)} T\right)\right) \\
& \simeq 4 \sigma_{\alpha}^{2} \delta(1-\delta)\left(1-\cos \left(2 \pi f_{\mathrm{AR}(2)} T\right)^{2}\right) .
\end{aligned}
$$

However, $\cos \left(2 \pi f_{\mathrm{AR}(2)} T\right)^{2} \simeq\left(1-\frac{\left(2 \pi f_{\mathrm{AR}(2)} T\right)^{2}}{2}\right)^{2} \simeq 1-\left(2 \pi f_{\mathrm{AR}(2)} T\right)^{2}$ since $\left(2 \pi f_{\mathrm{AR}(2)} T\right)^{4} \ll\left(2 \pi f_{\mathrm{AR}(2)} T\right)^{2} \ll 1$, so $(\mathrm{H} .4)$ leads to

$$
\sigma_{u}^{2} \simeq 4 \sigma_{\alpha}^{2} r(1-r)\left(2 \pi f_{\mathrm{AR}(2)} T\right)^{2},
$$

which is equivalent to $(28)$.

\section{References}

[1] A. H. El Husseini, E. P. Simon, L. Ros, Optimization of the second order autoregressive model AR(2) for Rayleigh-Jakes flat fading channel estimation with Kalman filter, in: Digital Signal Processing, IEEE, London, United Kingdom, 2017. 
[2] I. C. Msadaa, P. Cataldi, F. Filali, A comparative study between 802.11 p and mobile WiMAX-based V2I communication networks, in: Next Generation Mobile Applications, Services and Technologies (NGMAST), 2010 Fourth International Conference on, IEEE, 2010, pp. 186-191.

[3] R. H. Clarke, A statistical theory of mobile-radio reception, The Bell System Technical Journal 47 (6) (1968) 957-1000. doi:10.1002/j. 1538-7305.1968.tb00069.x.

[4] L. Lindbom, Simplified Kalman estimation of fading mobile radio channels: high performance at LMS computational load, in: IEEE International Conference on Acoust. Speech Signal Process., Vol. 3, 1993, pp. 352-355 vol.3. doi:10.1109/ICASSP. 1993.319507.

[5] G. Rajender, T. Anilkumar, K. S. Rao, Estimation and performance kalman based approach: A review, in: 2016 International Conference on Communication and Signal Processing (ICCSP), 2016, pp. 1145-1151. doi:10.1109/ICCSP.2016.7754332.

[6] M. Huang, X. Chen, L. Xiao, S. Zhou, J. Wang, Kalman-filter-based channel estimation for orthogonal frequency-division multiplexing systems in time-varying channels, IET communications 1 (4) (2007) 795801.

[7] S. Locubiche-Serra, G. Seco-Granados, J. A. Lopez-Salcedo, ClosedForm Approximation for the Convergence Time ofpth-Order Kalman Filters, IEEE Signal Processing Letters 25 (10) (2018) 1505-1509. doi:10.1109/LSP. 2018.2861210. 
[8] A. Jamoos, D. Labarre, E. Grivel, M. Najim, Two cross coupled kalman filters for joint estimation of mc-ds-cdma fading channels and their corresponding autoregressive parameters, in: 2005 13th European Signal Processing Conference, 2005, pp. 1-4.

[9] K. E. Baddour, N. C. Beaulieu, Autoregressive modeling for fading channel simulation, IEEE Transactions on Wireless Communications 4 (4) (2005) 1650-1662. doi:10.1109/TWC.2005.850327.

[10] M. Baruah, A. Misra, K. K. Sarma, AR-model of faded MIMO set-up optimized for ITU channels, in: 2015 2nd International Conference on Signal Processing and Integrated Networks (SPIN), 2015, pp. 242-247. doi:10.1109/SPIN.2015.7095294.

[11] P. Sharma, K. Chandra, Prediction of State Transitions in Rayleigh Fading Channels, IEEE Transactions on Vehicular Technology 56 (2) (2007) 416-425. doi:10.1109/TVT. 2007.891421.

[12] C. Komninakis, C. Fragouli, A. H. Sayed, R. D. Wesel, Multi-input multi-output fading channel tracking and equalization using Kalman estimation, IEEE Transactions on Signal Processing 50 (5) (2002) 10651076 .

[13] T. Y. Al-Naffouri, A. Paulraj, A forward-backward Kalman for the estimation of time-variant channels in OFDM, in: IEEE 6th Workshop on Signal Processing Advances in Wireless Communications, 2005., 2005, pp. 670-674. doi:10.1109/SPAWC.2005.1506224.

[14] B. Cheng, Yule-Walker Equations, Vol. 8, Wiley Online Library, 2005. 
[15] P. Banelli, R. C. Cannizzaro, L. Rugini, Data-Aided Kalman Tracking for Channel Estimation in Doppler-Affected OFDM Systems, in: 2007 IEEE International Conference on Acoustics, Speech and Signal Processing - ICASSP '07, Vol. 3, 2007, pp. III-133-III-136. doi: 10.1109/ICASSP.2007.366490.

[16] W. Chen, R. Zhang, Kalman-filter channel estimator for OFDM systems in time and frequency-selective fading environment, in: 2004 IEEE International Conference on Acoustics, Speech, and Signal Processing, Vol. 4, 2004, pp. iv-377-iv-380 vol.4. doi:10.1109/ICASSP.2004.1326842.

[17] M. S. Sohail, T. Y. Al-Naffouri, An EM based frequency domain channel estimation algorithm for multi-access OFDM systems, Signal Processing 90 (5) (2010) 1562-1572.

[18] W. Hassasneh, A. Jamoos, E. Grivel, H. A. Nour, Estimation of MC-DSCDMA Fading Channels Based on Kalman Filtering with High Order Autoregressive Models, in: 2006 Proceedings of the First Mobile Computing and Wireless Communication International Conference, 2006, pp. 145-149. doi:10.1109/MCWC.2006.4375212.

[19] R. Prasad, C. R. Murthy, B. D. Rao, Joint Approximately Sparse Channel Estimation and Data Detection in OFDM Systems Using Sparse Bayesian Learning, IEEE Transactions on Signal Processing 62 (14) (2014) 3591-3603. doi:10.1109/TSP.2014.2329272.

[20] V. Gutta, K. K. T. Anand, T. S. V. S. Movva, B. R. Korivi, S. Killamsetty, S. Padmanabhan, Low complexity channel estimation us- 
ing fuzzy Kalman Filter for fast time varying MIMO-OFDM systems, in: 2015 International Conference on Advances in Computing, Communications and Informatics (ICACCI), 2015, pp. 1771-1774. doi: 10.1109/ICACCI.2015.7275871.

[21] H. Abdzadeh-Ziabari, W. Zhu, M. N. S. Swamy, Joint Carrier Frequency Offset and Doubly Selective Channel Estimation for MIMOOFDMA Uplink With Kalman and Particle Filtering, IEEE Transactions on Signal Processing 66 (15) (2018) 4001-4012. doi:10.1109/ TSP.2018.2844222.

[22] A. Jamoos, M. Hussein, Estimation of UFMC Time-Varying Fading Channel Using Adaptive Filters, in: 2018 International Conference on Promising Electronic Technologies (ICPET), 2018, pp. 43-48. doi: 10.1109/ICPET.2018.00014.

[23] S. M. Shah, R. Samar, M. A. Z. Raja, Fractional-order algorithms for tracking Rayleigh fading channels, Nonlinear Dynamics 92 (3) (2018) $1243-1259$.

[24] O. P. Awe, Machine learning algorithms for cognitive radio wireless networks, Ph.D. thesis, (c) Olusegun Peter Awe (2015).

[25] E. P. Simon, L. Ros, H. Hijazi, M. Ghogho, Joint Carrier Frequency Offset and Channel Estimation for OFDM Systems via the EM Algorithm in the Presence of Very High Mobility, IEEE Transactions on Signal Processing 60 (2) (2012) 754-765. doi:10.1109/TSP. 2011.2174053. 
[26] H. Hijazi, L. Ros, Joint data QR-detection and Kalman estimation for OFDM time-varying Rayleigh channel complex gains, IEEE Transactions on Communications 58 (1) (2010) 170-178. doi:10.1109/TCOMM. 2010.01 .080296$.

[27] H. Abeida, J. . Brossier, L. Ros, J. Vila-Valls, An EM Algorithm for Path Delay and Complex Gain Estimation of Slowly Varying Fading Channel for CPM Signals, in: GLOBECOM 2009 - 2009 IEEE Global Telecommunications Conference, 2009, pp. 1-6. doi:10.1109/GLOCOM. 2009.5425719 .

[28] S. Ghandour-Haidar, L. Ros, J.-M. Brossier, On the use of first-order autoregressive modeling for Rayleigh flat fading channel estimation with Kalman filter, Signal Processing 92 (2) (2012) 601-606.

[29] A. Barbieri, A. Piemontese, G. Colavolpe, On the ARMA approximation for fading channels described by the Clarke model with applications to Kalman-based receivers, IEEE Transactions on Wireless Communications 8 (2) (2009) 535-540. doi:10.1109/TWC.2009.070188.

[30] H.-Y. Wu, A. Duel-Hallen, On the performance of coherent and noncoherent multiuser detectors for mobile radio CDMA channels, in: ICUPC, Vol. 1, 1996, pp. 76-80 vol.1. doi:10.1109/ICUPC.1996.557757.

[31] L. Lindbom, A. Ahlen, M. Sternad, M. Falkenstrom, Tracking of timevarying mobile radio channels. II. A case study, IEEE Transactions on Communications 50 (1) (2002) 156-167. doi:10.1109/26.975781. 
[32] B. C. Banister, J. R. Zeidler, Tracking performance of the RLS algorithm applied to an antenna array in a realistic fading environment, IEEE Transactions on Signal Processing 50 (5) (2002) 1037-1050. doi:10. $1109 / 78.995061$.

[33] L. Ros, E. P. Simon, Second-order modeling for Rayleigh flat fading channel estimation with Kalman Filter, in: 2011 17th International Conference on Digital Signal Processing (DSP), 2011, pp. 1-6. doi:10.1109/ICDSP.2011.6004912.

[34] S.-K. Chung, A phase tracking system for three phase utility interface inverters, IEEE Transactions on Power Electronics 15 (3) (2000) 431438. doi:10.1109/63.844502.

[35] R. Gerzaguet, L. Ros, J. Brossier, S. Ghandour-Haidar, F. Belveze, Selfadaptive stochastic rayleigh flat fading channel estimation, in: 2013 18th International Conference on Digital Signal Processing (DSP), 2013, pp. 1-6. doi:10.1109/ICDSP.2013.6622690.

[36] H. Shu, E. P. Simon, L. Ros, Third-order kalman filter: Tuning and steady-state performance, IEEE Signal Processing Letters 20 (11) (2013) 1082-1085. doi:10.1109/LSP.2013.2277668.

[37] D. Vaughan, A nonrecursive algebraic solution for the discrete Riccati equation, IEEE Transactions on Automatic Control 15 (5) (1970) 597599. doi:10.1109/TAC.1970.1099549.

[38] R. Gerzaguet, L. Ros, F. Belveze, J.-M. Brossier, Performance of a digital transmitter leakage LMS-based cancellation algorithm for multi- 
standard radio-frequency transceivers, Digital Signal Processing 51 (2016) 35-46.

[39] R. Gerzaguet, L. Ros, F. Belvèze, J.-M. Brossier, On the performance of digital adaptive spur cancellation for multi-standard radio frequency transceivers, Digital Signal Processing 33 (2014) 83-97.

[40] L. Lindbom, M. Sternad, A. Ahlen, Tracking of time-varying mobile radio channels .1. The Wiener LMS algorithm, IEEE Transactions on Communications 49 (12) (2001) 2207-2217. doi:10.1109/26.974267.

[41] S. Gazor, Prediction in LMS-type adaptive algorithms for smoothly time varying environments, IEEE Transactions on Signal Processing 47 (6) (1999) 1735-1739. doi:10.1109/78.765152.

[42] H. Shu, L. Ros, E. P. Simon, Simplified Random-Walk-Model-Based Kalman Filter for Slow to Moderate Fading Channel Estimation in OFDM Systems, IEEE Transactions on Signal Processing 62 (15) (2014) 4006-4017. doi:10.1109/TSP.2014.2332447.

[43] F. Golnaraghi, B. Kuo, Automatic control systems, Complex Variables $2(2010) 1-1$.

[44] H. L. V. Trees, K. L. Bell, Bayesian Bounds for Parameter Estimation and Nonlinear Filtering/Tracking, Wiley-IEEE Press, 2007.

[45] H. Hijazi, L. Ros, Bayesian CramerRao bounds for complex gain parameters estimation of slowly varying Rayleigh channel in OFDM systems, Signal Processing 89 (1) (2009) 111 - 115. doi:https://doi.org/10. 1016/j.sigpro.2008.07.017. 
[46] R. E. Blake, Basic vibration theory, Shock and vibration handbook 1 (1961) 2-8.

[47] B. Ekstrand, Analytical Steady State Solution for a Kalman Tracking Filter, IEEE Transactions on Aerospace and Electronic Systems AES19 (6) (1983) 815-819. doi:10.1109/TAES.1983.309393.

[48] R. Winkelstein, Closed form evaluation of symmetric two-sided complex integrals, communications Systems Research Section, 1981.

[49] A. H. el husseini, E. P. Simon, L. Ros, Approximation of $|1-L(z)|^{2}$, working paper or preprint (Oct. 2018).

URL https://hal . archives-ouvertes.fr/hal-01896043 